\title{
Differential Access to Price Information in Financial Markets
}

\author{
David Easley, Maureen O'Hara, and Liyan Yang*
}

\begin{abstract}
Recently, exchanges have been directly selling market data. We analyze how this practice affects price discovery, the cost of capital, return volatility, market liquidity, information production, and trader welfare. We show that selling price data increases the cost of capital and volatility, worsens market efficiency and liquidity, and discourages the production of fundamental information relative to a world in which all traders observe prices. Generally, allowing exchanges to sell price information benefits exchanges and harms liquidity traders. Overall, our results suggest that regulations on selling market data can play an important role in improving market quality and trader welfare.
\end{abstract}

\section{Introduction}

Improper early access to market data, even measured in milliseconds, can in today's markets be a real and substantial advantage that disproportionately disadvantages real and long-term traders. ${ }^{1}$

*Easley, dae3@cornell.edu, Cornell University, Department of Economics, Ithaca, NY 14853; O’Hara (corresponding author), mo19@cornell.edu, Cornell University, Johnson Graduate School of Management, Ithaca, NY 14853 and University of Technology Sydney; and Yang, liyan.yang@ rotman.utoronto.ca, University of Toronto, Rotman School of Management, Toronto, ON M5S 3E6, Canada and Peking University. We thank Hendrik Bessembinder (the editor) and Thierry Foucault (the referee) for constructive comments that have significantly improved the paper. We also thank the audiences at the 2011 Asian Finance Association Conference (Macao, China), the 2011 China International Conference in Finance (Wuhan, China), the 2011 Fields Institute 3C-Risk Forum (Toronto, Canada), the 2011 Financial Management Association European Conference (Porto, Portugal), the 2011 Northern Finance Association Conference (Vancouver, Canada), the 2011 Chinese Finance Association Best Paper Symposium (New York), and seminars in various institutions for their comments and suggestions. We thank the 2011 Northern Finance Association Conference and the 2011 Chinese Finance Association Best Paper Symposium for awarding the best-paper prizes to this paper. Yang thanks the Bank of Canada and Social Sciences and Humanities Research Council of Canada (SSHRC) for financial support. The views expressed herein are not necessarily those of the Bank of Canada and are the authors' alone.

${ }^{1}$ Robert Khuzami, director of the U.S. Securities and Exchange Commission's (SEC) Division of Enforcement, from "NYSE to Pay \$5 Million Penalty to SEC," Wall Street Journal (Sept. 14, 2012). 
Price information plays a crucial role in securities markets. Because of this fundamental role, securities regulators in the United States mandated the formation of a consolidated tape to provide real-time information on every trade execution in U.S. equity markets. Trade and quote data also provide a major source of revenue for stock exchanges. ${ }^{2}$ The U.S. exchanges jointly own the Consolidated Tape Association and the Consolidated Quote Association, which together sell the comprehensive quote and trade data available on the tape. Exchanges share in the tape revenue depending upon the volume of trades and the production of quotes in each market.

Some exchanges, however, have found a way to supplement their tape revenue by directly selling market data. For instance, the New York Stock Exchange (NYSE) sells a variety of direct data feeds via its products NYSE Best Quote, NYSE Open Book, and NYSE AMEX Best Quote; NASDAQ sells its trade and quote data directly under the product name NASDAQ ITCH; and the Hong Kong Exchange has experimented with provision of free real-time prices and is now moving to a system in which information vendors will be charged for data access. Purchasers of the data benefit in that they see the data before they appear on the consolidated tape, and exchanges benefit by essentially selling the same data twice, albeit at different speeds. How this practice affects the market is less clear.

In this paper we investigate what happens when some traders purchase early access to market information. In today's high-frequency markets, there are many instances where this occurs. First, purchasing fast data (along with practices such as co-location of trading terminals with exchange computers) gives rise to a practice called "latency arbitrage," whereby some traders are able to see market data before other traders. Kandel and Tkatch (2008), Hendershott and Moulton (2011), Hasbrouck and Saar (2013), and Easley, Hendershott, and Ramadorai (2014) analyze latency issues in equity markets. Second, in particularly volatile periods, delays in data transmission sometimes arise (the "Flash Crash" of May 6, 2010, is a case in point), and not all traders experience the same delay. ${ }^{3}$ Such data delays can also arise from exchange internal technology designs. ${ }^{4}$ Third, price data on some trades (odd-lot trades are a particularly important example) are produced by the market, but until recently were not provided to the public through the consolidated tape. ${ }^{5}$

\footnotetext{
${ }^{2}$ Stock exchange revenues come primarily from trading fees, listing fees, and market data. For the NYSE/Euronext, high market volumes resulted in market data revenues exceeding listing revenues in 2008 and 2009. In 2011, market data products contributed \$371 million to total NYSE revenue, and $\$ 333$ million to NASDAQ/OMX.

${ }^{3}$ The Summary Report of the Joint CFTC-SEC Advisory Committee on Emerging Regulatory Issues (p. 76) noted also that at the height of the flash crash, NYSE quotes in 1,665 securities had average delays to the Consolidated Quotation System (CQS) of over 10 seconds. Between 2:45 and 2:50PM, over 40 of these stocks had average delays greater than 20 seconds. See also Peterson (2010).

${ }^{4}$ The SEC recently fined NYSE/Euronext $\$ 5$ million for sending market data to proprietary traders before sending such data to the consolidated tape and quote feeds. The NYSE claimed "that the timing differential stemmed from technology issues" (see "NYSE to Pay 5 Million Dollar Fine to SEC," Wall Street Journal (Sept. 14, 2012)).

${ }^{5}$ O'Hara, Yao, and Ye (2014) demonstrate that odd lots play an increasingly important role in high-frequency markets. As suggested in that paper, the SEC required reporting of odd lots to the consolidated tape as of Dec. 9, 2013.
} 
Our goal is to understand how differential access to price information affects traders' behavior and the performance of the market. We cast our analysis in a model in which rational traders trade a risky asset with liquidity traders. Rational traders can either produce or buy fundamental information as well as purchasing price data from a for-profit exchange to facilitate their trading. If the profit-maximizing price of market data is high, then in equilibrium only some traders purchase price data, and therefore our model exhibits differential access to price information. We show that differential access generally increases the cost of capital and volatility, reduces market efficiency and liquidity, discourages the production of fundamental information, harms liquidity traders, and benefits rational traders relative to an economy in which all traders observe price data simultaneously.

The negative effect on price discovery and the positive effects on the cost of capital and on volatility arise because "price-informed" traders benefit at the expense of "price-uninformed" traders who in turn scale back their holdings to offset the increased risk arising from their lack of information about prices. The negative effect on market liquidity arises because "price-informed" traders make prices more responsive to fundamentals and thus less responsive to noise trading. Relative to a world in which all traders observe prices, allowing exchanges to sell price information enhances exchange profit and benefits rational traders at the expense of liquidity traders, whose welfare is increasing in liquidity and decreasing in the equity risk premium.

Once the impact of selling price data on fundamental information is taken into account, these negative effects on market quality become even stronger. We show that acquiring fundamental data and purchasing price data are complementary. In our economy, traders do not necessarily observe the price (before trading), and because price information and fundamental information are jointly useful in predicting the fundamental value of the asset, traders acquire both fundamental data and price data simultaneously rather than separately. As a result, if the equilibrium price of market data is high, which directly lowers traders' incentives to acquire price information, the amount of fundamental information produced decreases as well, thereby further harming price informativeness relative to an economy without differential access. This, in turn, increases both the cost of capital and return volatility, and it typically lowers liquidity.

A number of authors (e.g., Admati and Pfleiderer (1986), (1988), (1990), Allen (1990), Fishman and Hagerty (1995), Veldkamp (2006), García and Vanden (2009), García and Sangiorgi (2011), and Chen and Wilhelm (2012)) consider the issue of selling information in financial markets, although typically in the context of analysts selling fundamental information to other traders. We extend this literature by showing how price data and fundamental data are complementary in our setting. This contrasts with the central result highlighted by the information sales literature (e.g., Admati and Pfleiderer (1986), García and Sangiorgi (2011)), which states that in traditional settings, information on the price tends to be a substitute for fundamental information. Our analysis further complements this literature by exploring the implications of selling price data for market outcomes (i.e., market efficiency, the cost of capital, return volatility, and liquidity), an issue not addressed by earlier literature. Finally, in our analysis the precision of the 
price data being sold by the exchange is endogenous because it is determined in equilibrium by the amount of private fundamental information, which, in turn, is affected by the equilibrium precision of the price data. By contrast, in the earlier literature the precision of the to-be-sold data is exogenous (in both cases the value of the information is endogenous).

Another large literature examines differential access to information in the context of insider trading (see, e.g., Glosten (1989), Fishman and Hagerty (1990), Leland (1992), and Cao, Field, and Hanka (2004)). In our baseline analysis, all traders have equal access to information regarding underlying asset values, but some traders have "inside information" about market data. In common with the insider trading literature, we find that this form of differential information affects liquidity, price discovery, and the cost of capital. Our finding that greater availability of price data lowers the cost of capital complements Leland's (1992) finding that more insider trading can have a similar positive effect in the market.

A recent paper by Cespa and Foucault (2014) also investigates the role of price data in securities markets. Their analysis focuses on explaining why a forprofit exchange optimally restricts access to price information and how this practice affects price discovery. In contrast, our analysis explores broader implications of selling price information for market quality beyond market efficiency (such as the cost of capital, liquidity, and return volatility), which is critical for the current policy debate. Most importantly, we study the interaction between selling price information and gathering private fundamental information, which has been largely ignored in the existing literature. Because of the various feedback effects between price information and fundamental information, analyzing this interaction yields new theoretical results. For example, we show that purchasing price information and acquiring fundamental information are complementary, and, as a result, curbing access to price information typically reduces the amount of fundamental information produced in equilibrium, which further harms market quality. Hence, our paper complements the analysis by Cespa and Foucault (2014).

Our analysis yields insights into the desirability of allowing exchanges to sell data, an issue that is now a major policy debate both in the United States and in Europe. In Europe, there is no consolidated tape, so exchange-data products give traders information not available elsewhere. In the United States, exchange-data products give traders information before it appears on the tape. Given the speed at which high-frequency trading now occurs, any delay now matters for the market equilibrium. ${ }^{6}$ We develop the implications of our research for this debate and our recommendations for regulatory policy in more detail later in the paper.

\section{The Model}

There are two tradable assets in our economy: one risk-free asset, cash, which has a constant value of 1 ; and one risky asset, which has a price of $\tilde{p}$ per unit and

\footnotetext{
${ }^{6}$ Direct evidence on the importance of speed is starkly illustrated by the construction of the Hibernian Express, the first new transatlantic cable in 10 years. When it was completed in 2013, the new $\$ 300$ million cable reduced the speed of transmitting orders between London and New York to 59.6 milliseconds from 64.8 milliseconds. The project was privately funded and its customers were large hedge funds engaged in high-frequency trading.
} 
an uncertain future value denoted $\tilde{v}$. We assume that $\tilde{v} \sim N\left(\bar{v}, 1 / \rho_{v}\right)$ with $\bar{v}>0$ and $\rho_{v}>0$.

There are two types of traders: rational traders and liquidity traders. There is a continuum $[0,1]$ of rational traders with constant absolute risk aversion (CARA) utility functions with a common coefficient of risk aversion $\gamma>0$. Each rational trader is endowed with cash only, and for simplicity we suppose that their endowment is 0 . Liquidity traders provide the randomness necessary to make our rational expectations equilibrium partially revealing in the sense that they supply $\tilde{x}$ units of the risky asset per capita to the market. We do not endogenize the behavior of liquidity traders; rather, we view them as individuals who are trading to invest new cash flows or to liquidate assets to meet unexpected consumption needs. ${ }^{7}$

We assume that $\tilde{x} \sim N\left(\bar{x}, 1 / \rho_{x}\right)$ with $\bar{x}>0$ and $\rho_{x}>0$. The assumption that the mean per capita supply of the asset is positive is important for our results. If instead it is 0 , then on average there is no aggregate risk to be borne, and in equilibrium no one will be rewarded for bearing it. We believe that the pricing of aggregate risk is important, so we focus on the case in which it exists. One can view this risky asset as a proxy for the stock market, and in this case aggregate uncertainty is unavoidable.

Initially we assume that each trader $i \in[0,1]$ is endowed with an independent private signal: ${ }^{8}$

$$
\begin{aligned}
\tilde{s}_{i} & =\tilde{v}+\tilde{\varepsilon}_{i}, \quad \text { with } \tilde{\varepsilon}_{i} \sim N\left(0,1 / \rho_{\varepsilon}\right) \text { and } \\
\rho_{\varepsilon} & >0 .
\end{aligned}
$$

In Section $\mathrm{V}$, we endogenize the decision to acquire private signals.

Rational traders are further categorized into two groups according to whether they pay the profit-maximizing exchange a price of $q>0$ to observe the current stock price $\tilde{p}$. If a trader acquires $\tilde{p}$, then he or she can submit orders conditional on $\tilde{p}$ and $\tilde{s}_{i}$ : $\mathrm{DI}\left(\tilde{p}, \tilde{s}_{i}\right)$. If a trader does not acquire $\tilde{p}$, then he or she can only submit orders conditional on $\tilde{s}_{i}$ : DU $\left(\tilde{s}_{i}\right)$. We call those traders purchasing $\tilde{p}$ price-informed traders; the traders not purchasing $\tilde{p}$ are called price-uninformed traders. We suppose that there is a fraction $\mu>0$ of price-informed traders. In Section III.C, we endogenize the decision to acquire the contemporaneous price.

It is worth pointing out that in our model the exchange is selling access to contemporaneous price information. Traders who purchase this information can condition their demand for the risky asset on the equilibrium price, whereas those who do not purchase access to price information cannot condition on the contemporaneous price. This is of course an abstraction from how modern stock markets function. In reality, no one can condition on the equilibrium price (and the fact that it is an equilibrium), and actually there is no single equilibrium price for a stock.

\footnotetext{
${ }^{7}$ The noise induced by liquidity traders equivalently can be viewed as random (from the point of view of rational traders) float of securities. Alternatively, it is possible to model the decision problem of traders who experience endowment shocks, which would endogenize our liquidity traders. We do not do this, because it greatly complicates the analysis without providing additional insights.

${ }^{8}$ For a foundation for a continuum of independent random variables and an exact version of the law of large numbers, see Judd (1985).
} 
We use this abstraction to provide insight into the effects of selling differential access to price information in the cleanest possible setting. We discuss how this abstraction affects the interpretation of our results in Section VI.B.

Our model is designed to capture the multiple feedback effects arising from selling price information: The precision of price information affects the incentive of traders to purchase this information, and the trading of the resulting priceinformed traders will, in turn, determine the precision of the price information they purchase; in addition, the incentive of traders to acquire fundamental information is affected, and this too affects the precision of price information. An alternative would be to examine these feedback effects in a fully dynamic model in which the exchange sells price data formed in previous periods. Constructing and analyzing such a dynamic model is a daunting task. We believe that our 1-period model is a good approximation to capturing the impact of selling price data. ${ }^{9}$

\section{Equilibrium Characterization}

\section{A. The Equilibrium at the Trading Stage}

We begin by deriving a rational expectations equilibrium (REE) in the stock market with a fixed fraction $\mu$ of price-informed traders. In an equilibrium, there is a price function such that if all traders conjecture that prices are determined by this function, then market-clearing prices are in fact determined by this function. The functional form we derive is:

$$
\tilde{p}=\alpha+\beta \tilde{v}-\lambda \tilde{x} .
$$

So, observing the price is equivalent to observing the following signal about the asset payoff $\tilde{v}$ :

$$
\tilde{s}_{p}=\frac{\tilde{p}-\alpha+\lambda \bar{x}}{\beta}=\tilde{v}-m^{-1}(\tilde{x}-\bar{x}),
$$

where

$$
m=\frac{\beta}{\lambda}
$$

denotes the "market efficiency" or "price informativeness" measure, as is standard in noisy REE models (e.g., Kyle (1989), Peress (2010), and Ozsoylev and Walden (2011)).

Given the CARA-normal setup, the demand functions and indirect utility functions for the traders in our economy take on standard forms. For priceinformed traders and price-uninformed traders these functions are, respectively:

$$
\begin{aligned}
\operatorname{DI}\left(\tilde{p}, \tilde{s}_{i}\right) & =\frac{\mathrm{E}\left(\tilde{v} \mid \tilde{p}, \tilde{s}_{i}\right)-\tilde{p}}{\gamma \operatorname{var}\left(\tilde{v} \mid \tilde{p}, \tilde{s}_{i}\right)}, \\
\operatorname{VI}\left(\tilde{p}, \tilde{s}_{i}\right) & =-\exp \left\{-\gamma \bar{W}_{i}+\gamma q-\frac{\left[\mathrm{E}\left(\tilde{v}-\tilde{p} \mid \tilde{p}, \tilde{s}_{i}\right)\right]^{2}}{2 \operatorname{var}\left(\tilde{v} \mid \tilde{p}, \tilde{s}_{i}\right)}\right\},
\end{aligned}
$$

\footnotetext{
${ }^{9}$ Our paper is similar in spirit to that of Biais, Foucault, and Moinas (2015), who use a 1-period setting to analyze high-frequency trading.
} 


$$
\begin{aligned}
\operatorname{DU}\left(\tilde{s}_{i}\right) & =\frac{\mathrm{E}\left(\tilde{v}-\tilde{p} \mid \tilde{s}_{i}\right)}{\gamma \operatorname{var}\left(\tilde{v}-\tilde{p} \mid \tilde{s}_{i}\right)}, \\
\operatorname{VU}\left(\tilde{s}_{i}\right) & =-\exp \left\{-\gamma \bar{W}_{i}-\frac{\left[\mathrm{E}\left(\tilde{v}-\tilde{p} \mid \tilde{s}_{i}\right)\right]^{2}}{2 \operatorname{var}\left(\tilde{v}-\tilde{p} \mid \tilde{s}_{i}\right)}\right\},
\end{aligned}
$$

where $\bar{W}_{i}$ is the initial wealth of trader $i$.

The market-clearing condition is:

$$
\int_{0}^{\mu} \mathrm{DI}\left(\tilde{p}, \tilde{s}_{i}\right) d i+\int_{0}^{1-\mu} \mathrm{DU}\left(\tilde{s}_{i}\right) d i=\tilde{x},
$$

which requires per capita demand for the risky asset to equal per capita supply. To derive the equilibrium price function, we use Bayes's rule to compute the conditional moments in the demand functions of various traders, then use the marketclearing condition in equation (8) to express the price in terms of the fundamental $\tilde{v}$ and the noise supply $\tilde{x}$, and finally compare with the conjectured price function in equation (1) to obtain a system defining the two unknown coefficients $\beta$ and $\lambda$. Solving this system yields the following proposition characterizing the equilibrium price function:

Proposition 1. Suppose $\mu>0$. There exists a unique partially revealing REE, with price function

$$
\tilde{p}=\alpha+\beta \tilde{v}-\lambda \tilde{x},
$$

where

$$
\begin{aligned}
\beta= & \frac{\gamma \mu^{-1} m+m^{2} \rho_{x}}{\rho_{v}+\rho_{\varepsilon}+m^{2} \rho_{x}}, \\
\lambda= & \frac{\gamma \mu^{-1}+m \rho_{x}}{\rho_{v}+\rho_{\varepsilon}+m^{2} \rho_{x}}, \\
\alpha= & (1-\beta) \bar{v}+\lambda \bar{x} \\
& -\gamma \bar{x}\left[\mu \operatorname{var}^{-1}\left(\tilde{v} \mid \tilde{p}, \tilde{s}_{i}\right)+(1-\mu) \operatorname{var}^{-1}\left(\tilde{v}-\tilde{p} \mid \tilde{s}_{i}\right)\right]^{-1},
\end{aligned}
$$

with

$$
\begin{aligned}
m & =\frac{\mu \rho_{\varepsilon}\left(\gamma^{2}+\mu \rho_{v} \rho_{x}+\mu \rho_{\varepsilon} \rho_{x}\right)}{\gamma\left(\gamma^{2}+\mu^{2} \rho_{v} \rho_{x}+\mu \rho_{\varepsilon} \rho_{x}\right)} \\
\frac{1}{\operatorname{var}\left(\tilde{v}-\tilde{p} \mid \tilde{s}_{i}\right)} & =\frac{\mu^{2} \rho_{x}\left(\rho_{v}+\rho_{\varepsilon}\right)}{\gamma^{2}+\mu^{2} \rho_{x}\left(\rho_{v}+\rho_{\varepsilon}\right)} \frac{1}{\operatorname{var}\left(\tilde{v} \mid \tilde{p}, \tilde{s}_{i}\right)}, \\
\frac{1}{\operatorname{var}\left(\tilde{v} \mid \tilde{p}, \tilde{s}_{i}\right)} & =\rho_{v}+\rho_{\varepsilon}+m^{2} \rho_{x} .
\end{aligned}
$$

For an equilibrium to exist, we need to assume $\mu>0$ in Proposition 1 . This is because when $\mu=0$, the market clearing price is not well defined, because no trader's demand is sensitive to the price. The assumption of $\mu>0$ is consistent with the full equilibrium that we analyze in Section III.C, where $\mu$ is endogenously derived from the exchange's profit-maximization behavior. That is, the 
profit-maximizing exchange will always set the cost of price information so that $\mu>0$, since if $\mu=0$, its profit is zero too. Of course, from a theoretical perspective, the case of $\mu=0$ may be of its own interest, because it corresponds to an economy with no price information sold at all. We can examine the behavior of variables as $\mu \rightarrow 0$ in our model to approximate such an economy.

We define the cost of capital (CC), return volatility (RET_VOL), and market liquidity (LIQUIDITY), as usual, as:

$$
\begin{aligned}
\mathrm{CC} & \equiv \mathrm{E}(\tilde{v}-\tilde{p}), \\
\text { RET_VOL } & \equiv \sigma(\tilde{v}-\tilde{p}), \quad \text { and } \\
\text { LIQUIDITY } & \equiv \lambda^{-1} .
\end{aligned}
$$

That is, the $\mathrm{CC}$ is the expected difference between the cash flow generated by the risky asset and its price. This difference arises from the compensation required to induce rational traders to hold the risky asset. The return on the risky asset is $(\tilde{v}-\tilde{p})$, and thus its volatility can be measured by $\sigma(\tilde{v}-\tilde{p})$. Market liquidity measures the market depth: A smaller $\lambda$ means that liquidity trading has a smaller price impact, and so the market is deeper. Much of the positive analysis in our baseline model is focused on the effect of the sale of price information on these three statistics and on price discovery (i.e., price informativeness $m$ ). In Section V, we also examine how selling price data affect the production of fundamental information.

\section{B. The Impact of the Fraction of Price-Informed Traders}

1. Price Informativeness

Taking the derivative with respect to $\mu$ in equation (11) in Proposition 1, we have

$$
\begin{aligned}
\frac{\partial m}{\partial \mu} & =\left(\frac{\rho_{\varepsilon}}{\gamma}\right) \frac{\left(\gamma^{2}+\mu \rho_{\varepsilon} \rho_{x}\right)^{2}+\gamma^{2} \mu(2-\mu) \rho_{v} \rho_{x}+\mu^{2} \rho_{v} \rho_{\varepsilon} \rho_{x}^{2}}{\left(\gamma^{2}+\mu^{2} \rho_{v} \rho_{x}+\mu \rho_{\varepsilon} \rho_{x}\right)^{2}} \\
& >0 .
\end{aligned}
$$

Thus, increasing the fraction of price-informed traders improves price informativeness. This is because price-informed traders trade more aggressively on their fundamental information than do price-uninformed traders, so more priceinformed traders will bring more information into the equilibrium price.

Corollary 1. As more traders become price-informed traders, the price becomes more informative; that is, $\partial m / \partial \mu>0$.

2. The Cost of Capital

By Proposition 1, the cost of capital is

$$
\begin{aligned}
\mathrm{CC} & \equiv \mathrm{E}(\tilde{v}-\tilde{p}) \\
& =\frac{\gamma \bar{x}}{\mu \operatorname{var}^{-1}\left(\tilde{v} \mid \tilde{p}, \tilde{s}_{i}\right)+(1-\mu) \operatorname{var}^{-1}\left(\tilde{v}-\tilde{p} \mid \tilde{s}_{i}\right)} .
\end{aligned}
$$

So, the cost of capital is increasing in traders' risk aversion $\gamma$, the asset supply $\bar{x}$, and the average risk that traders are exposed to per unit of the asset $\left[\mu \operatorname{var}^{-1}\left(\tilde{v} \mid \tilde{p}, \tilde{s}_{i}\right)+(1-\mu) \operatorname{var}^{-1}\left(\tilde{v}-\tilde{p} \mid \tilde{s}_{i}\right)\right]^{-1}$. 
Increasing the fraction of price-informed traders reduces the cost of capital through two effects, one direct and one indirect. The direct effect occurs because price-informed traders demand more of the risky asset than price-uninformed traders, because their knowledge of the price reduces the riskiness of the asset for them. So, as more traders become price-informed traders, the equilibrium price of the risky asset increases. The indirect effect occurs through the positive impact of a more informative price on the demands of all traders. For price-informed traders, a more informative price helps them to more accurately forecast the future asset value, reducing their risk of trading the risky asset. For price-uninformed traders, a more informative price implies that the prevailing price is closer to the fundamental of the asset, and hence they, too, face less risk. Thus, a more informative price causes all traders to demand more of the risky asset, thereby increasing its price. This discussion is summarized in the following corollary:

Corollary 2. As more traders become price-informed traders, the cost of capital decreases; that is, $\partial C C / \partial \mu<0$.

Note that this result is independent of the value of $\mu$. In particular, when $\mu$ is very low, it is mainly the direct effect at work, because there is little information in the price (because $m$ is close to 0 ). Still, price-informed traders face no price risk and trade more aggressively than price-uninformed traders, and so increasing the fraction $\mu$ of price-informed traders will push prices up on average, lowering the cost of capital.

\section{Return Volatility}

As we increase the fraction of price-informed traders, the price will reveal more information about $\tilde{v}$, causing the difference between the future value of the asset and its current price to be smaller for any amount of noise in the economy. Thus, the return on the risky asset, $(\tilde{v}-\tilde{p})$, is smaller, which reduces return volatility.

Corollary 3. As more traders become price-informed traders, return volatility decreases; that is, $\partial \mathrm{RET}_{\text {_VOL }} / \partial \mu<0$.

4. Liquidity

By equation (10) in Proposition 1,

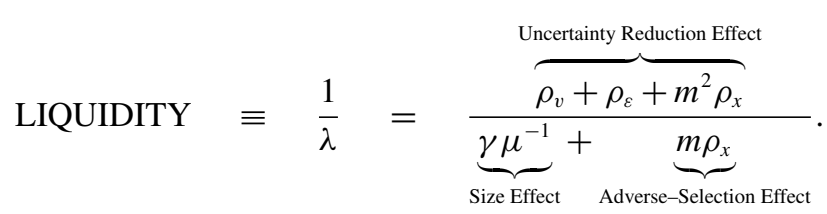

The fraction of price-informed traders, $\mu$, affects each of the three components of liquidity. The first effect is a direct effect arising from the term $\left(\gamma \mu^{-1}\right)$ in the denominator. We call this the "size effect" because it describes the direct impact of the size of the fraction of the price-informed trader population on liquidity. An increase in $\mu$ will positively affect liquidity through this effect. When $\mu$ is small, the price will be very responsive to liquidity trading $\tilde{x}$; that is, the 
market will be illiquid, because changes in liquidity trader demand will have to be absorbed by the few price-informed traders, and this can occur only through large price changes.

The other effects on liquidity are indirect effects that occur through price informativeness $m$. Changes in $\mu$ affect liquidity differently through these two effects. The "uncertainty reduction effect" captures the fact that increasing $\mu$ will increase price informativeness, causing price-informed traders to trade more aggressively, making price more responsive to fundamentals $\tilde{v}$ than to liquidity trading $\tilde{x}$, and thus improving liquidity (see Goldstein and Yang (2015)). In contrast, the "adverse-selection effect" captures the fact that the improved price informativeness will make it possible for price-informed traders to draw stronger inferences from price, making their demands more responsive to price changes induced by liquidity trading, and thus making the market less liquid.

Although the impact of $\mu$ on liquidity is complex, we can show that the positive effects dominate, so that as the fraction of price-informed traders increases, market liquidity increases.

Corollary 4. As more traders become price-informed traders, the market becomes more liquid. That is, $\partial$ LIQUIDITY $/ \partial \mu>0$.

Figure 1 illustrates Corollaries $1-4$ for the parameter values given by Table 1 . We set the risk-aversion parameter to be $\gamma=3$. The other parameters in Table 1 are borrowed from Leland's (1992) calibration. ${ }^{10}$ The expected payoff of the risky asset $\bar{v}$ is normalized to 1 . The ex ante payoff precision $\rho_{v}$ is 25 , which gives an annual volatility of $20 \%$. We follow Gennotte and Leland (1990) in setting the rational trader's signal-to-noise ratio as 0.2 (i.e., $\rho_{\varepsilon} / \rho_{v}=0.2$ ), implying that the precision of the private signal is $\rho_{\varepsilon}=5$. We normalize the per capita supply of the risky asset to 1 , so that $\bar{x}=1$. The precision of the liquidity trading is set to 4; that is, $\rho_{x}=4$, which corresponds to an annual volatility of liquidity trading equal to $50 \%$ of total supply. We see that, consistent with Corollaries $1-4$, as the

TABLE 1

Parameter Values

Table 1 presents parameter values that are used to compute the equilibrium outcomes in our numerical analysis.

Parameters

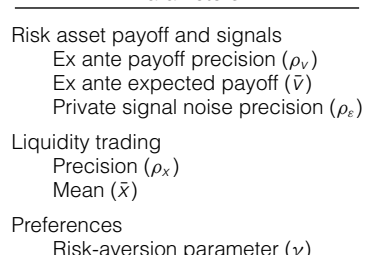

Risk-aversion parameter $(\gamma)$ $\underline{\text { Values }}$

25

5

4

1

3

\footnotetext{
${ }^{10}$ Because Leland's (1992) original calibration is based on annual Standard \& Poor's (S\&P) 500 data, our results should also be interpreted on an annual basis. As we explained in the Introduction, the delay of price data in reality (and hence the period length in our model) depends on specific scenarios, varying from milliseconds to infinity. We chose an annual frequency for simplicity.
} 
FIGURE 1

The Impact of Observing Price Information on Market Outcomes

Figure 1 plots price informativeness, the cost of capital, return volatility, and liquidity as a function of the fraction of price-informed traders. All other parameter values are fixed at the values in Table 1.
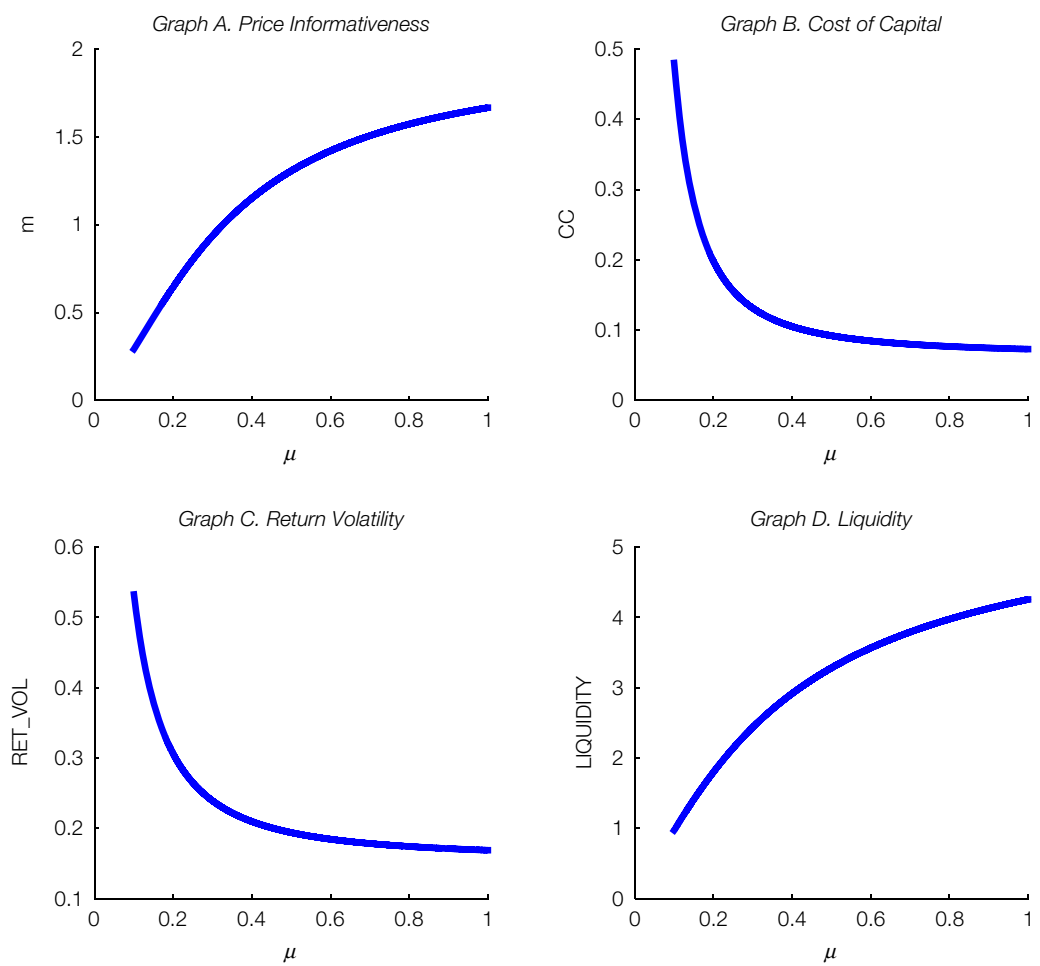

fraction of price-informed traders increases, price informativeness and liquidity increase in Graphs A and D, and the cost of capital and return volatility decrease in Graphs B and C.

\section{Buying and Selling Price Information}

The equilibrium fraction of traders who purchase price information is determined by comparing the indirect utility of a price-informed trader with that of a price-uninformed trader. The benefit, $B(\mu)$, of purchasing price data is the difference between the certainty equivalents of these two indirect utilities. ${ }^{11}$ Calculation shows that this benefit is:

$$
\begin{aligned}
B(\mu) & =\frac{1}{2 \gamma} \ln \left[\frac{\operatorname{var}\left(\tilde{v}-\tilde{p} \mid \tilde{s}_{i}\right)}{\operatorname{var}\left(\tilde{v}-\tilde{p} \mid \tilde{p}, \tilde{s}_{i}\right)}\right] \\
& =\frac{1}{2 \gamma} \ln \left[1+\frac{\gamma^{2}}{\mu^{2} \rho_{x}\left(\rho_{v}+\rho_{\varepsilon}\right)}\right] .
\end{aligned}
$$

\footnotetext{
${ }^{11}$ This is the difference between $\phi_{p s}$ and $\phi_{s}$ in equations (26) and (28) introduced in Section V.
} 
Observing the market-clearing price has two potential benefits: The trader knows the prevailing price at which his or her trade will execute, and hence the immediate price risk is reduced; and, the price contains information about the future fundamental value $\tilde{v}$, and hence it is useful for forecasting. The first benefit tends to make $B(\mu)$ increasing in $\mu$ : As more traders observe the price, the price becomes more efficient, and thus more valuable. The second benefit tends to make $B(\mu)$ decreasing in $\mu$ : An increase in $\mu$ will cause the equilibrium price to be closer to the fundamental value, thus decreasing the benefit of reducing price risk (recall that price informativeness increases with $\mu$ ). Overall, as the second equality in equation (16) shows, the second effect dominates, so that the benefit $B(\mu)$ of observing price information decreases with the fraction $\mu$ of price-informed traders.

For any given $\mu$, the maximum amount that the exchange can charge each of these traders to observe the price is the benefit they receive, $B(\mu)$. The revenue the exchange receives if fraction $\mu$ is price-informed is thus $B(\mu) \mu$. The exchange's decision problem is:

$$
\max _{\mu \in(0,1]} B(\mu) \mu=\frac{1}{2 \gamma} \max _{\mu}\left\{\mu \ln \left[1+\frac{\gamma^{2}}{\mu^{2} \rho_{x}\left(\rho_{v}+\rho_{\varepsilon}\right)}\right]\right\} .
$$

In this analysis, we have assumed that the exchange only derives revenues from the sale of price information. In reality exchanges also derive revenues from a variety of sources, including trading fees. We have ignored this fact by implicitly assuming that changing $\mu$ does not affect revenues from trading that much. Fully incorporating this linkage would significantly complicate the analysis.

There are two possibilities for the optimal (from the point of view of the exchange) $\mu^{*}: \mu^{*}=1$ or $0<\mu^{*}<1$. The following proposition provides a characterization of the parameters for which it is optimal for the exchange to sell price information to everyone (i.e., $\mu^{*}$ is 1 ):

Proposition 2. Let $\hat{z}$ be the constant solving the equation $\ln (1+\hat{z})=(2 \hat{z}) /(1+\hat{z})$. If $\gamma^{2} /\left[\rho_{x}\left(\rho_{v}+\rho_{\varepsilon}\right)\right] \geq \hat{z}$, the equilibrium fraction of price-informed traders is 1 (i.e., $\left.\mu^{*}=1\right)$, and the equilibrium price of information is $q^{*}=B(1)$. Otherwise, the optimal solution is $\mu^{*}=\gamma / \sqrt{\hat{z} \rho_{x}\left(\rho_{v}+\rho_{\varepsilon}\right)}$, and the equilibrium price of information is $q^{*}=\ln (1+\hat{z}) /(2 \gamma)=\hat{z} /[\gamma(1+\hat{z})]$.

Proposition 2 shows that when the risk-aversion parameter $\gamma$ is sufficiently large, the exchange will set $\mu^{*}=1$. In this case, price information is so valuable to traders that the exchange can sell it to everyone at a high price. Under the technology parameter configuration in Table 1, the lower bound for $\gamma$ characterized by Proposition 2 is equal to 21.69. Figure 2 provides an illustration of this result. In Graph A, $\gamma$ is equal to 3, which is relatively small, and the optimal fraction of price-informed traders is interior, $\mu^{*}=0.1383$. In Graph $\mathrm{B}, \gamma$ is equal to 25 , which exceeds the lower bound 21.69 , and thus the profit-maximizing fraction $\mu^{*}$ of price-informed traders is 1 .

\section{The Impact of Selling Price Information}

We now evaluate the effects of allowing the exchange to sell access to the price rather than requiring that it be provided to everyone for free. We consider 
FIGURE 2

Exchange Profit in Economies with Exogenous Fundamental Information

Figure 2 plots the profit of the exchange as a function of the fraction of price-informed traders when each rational trader is endowed with fundamental information. All other parameter values are fixed at the values in Table 1.
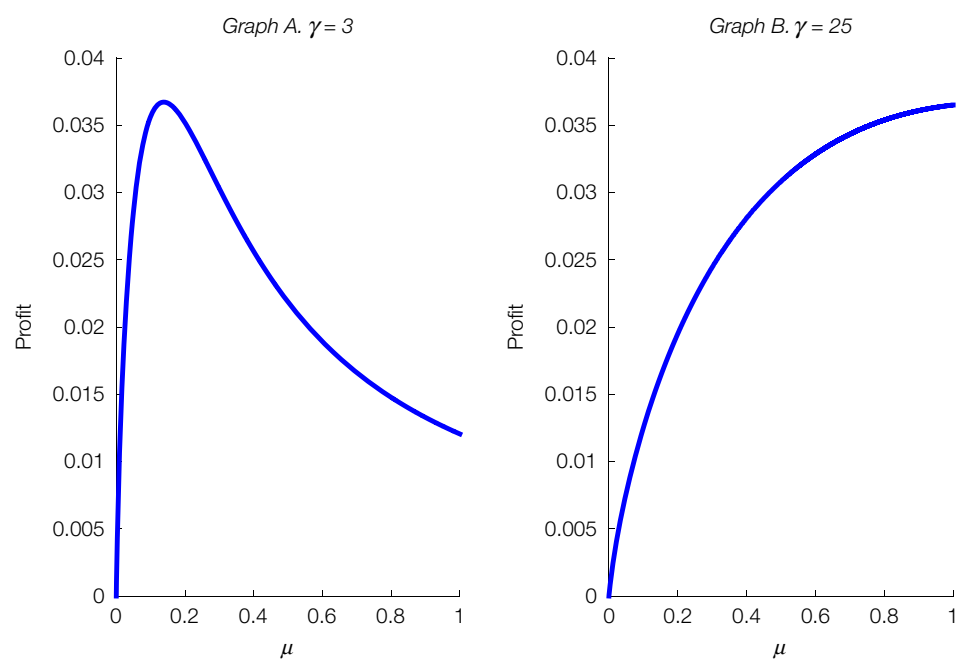

two alternative economies. In ECONOMY ${ }^{\mathrm{F}}$ all traders can freely observe the price perhaps because of SEC regulations requiring equal access to market prices. We caution that one should not literally interpret this ECONOMY ${ }^{\mathrm{F}}$ as an economy with free market data. Instead, a more proper interpretation is that ECONOMYF represents an economy with a relatively low price $q$ for market data. This is because our model does not capture all reasons for exchanges to charge for price data (e.g., producing market data is costly). We will discuss this issue further in Section VI.B. Here, in order to convey our ideas most effectively, we take an extreme view and set $q=0$ in ECONOMY ${ }^{\mathrm{F}}$.

ECONOMY ${ }^{\mathrm{D}}$ is the economy analyzed in the previous sections in which traders are differentially informed, and the exchange chooses the profitmaximizing price to charge for access to market prices. The consequences of requiring the exchange to provide free access are not obvious because market prices will differ in the two economies. At this point in the analysis we keep access to private signals about the value of the risky asset exogenous. Of course, selling access to market prices affects the value of private information about the risky asset, and in the next section we endogenize the decision to acquire private fundamental information.

\section{A. Benchmark Economy: ECONOMYF}

We use ECONOMY ${ }^{\mathrm{F}}$ in which all traders can freely observe the equilibrium price as the benchmark economy. This benchmark economy can be viewed as an economy in which the exchange is required to set the cost, $q$, of observing the market price to (be sufficiently close to) 0 . In this case all traders have price information (i.e., $\mu=1$ ). 
The equilibrium price of the risky asset $\tilde{p}^{F}$ is the equilibrium price in Proposition 1 with $\mu=1$. The welfare of each rational trader is the certainty equivalent of their ex ante utility:

$$
\mathrm{CE}^{F}=-\frac{1}{\gamma} \ln \left(-\mathrm{E}\left[\mathrm{VI}\left(\tilde{p}^{F}, \tilde{s}_{i}\right)\right]\right),
$$

where

$$
\mathrm{E}\left[\mathrm{VI}\left(\tilde{p}^{F}, \tilde{s}_{i}\right)\right]=-\sqrt{\frac{\operatorname{var}\left(\tilde{v}-\tilde{p}^{F} \mid \tilde{p}^{F}, \tilde{s}_{i}\right)}{\operatorname{var}\left(\tilde{v}-\tilde{p}^{F}\right)}} \exp \left\{-\frac{\left[\mathrm{E}\left(\tilde{v}-\tilde{p}^{F}\right)\right]^{2}}{2 \operatorname{var}\left(\tilde{v}-\tilde{p}^{F}\right)}\right\} .
$$

The revenue that a liquidity trader receives from selling $\tilde{x}$ is $\left(\tilde{p}^{F}-\tilde{v}\right) \tilde{x}$. The expected revenue $\mathrm{E}\left[\left(\tilde{p}^{F}-\tilde{v}\right) \tilde{x}\right]$ is thus the negative of the expected opportunity cost $\mathrm{E}\left[\left(\tilde{v}-\tilde{p}^{F}\right) \tilde{x}\right]$ associated with a trade of $\tilde{x}$ shares. We do not have a utility function for liquidity traders, and so we cannot compute their welfare. Instead, we use expected revenue as a proxy for the welfare of liquidity traders to capture the idea that they are better off if they can realize their hedging or liquidity needs at a lower expected opportunity cost. ${ }^{12}$ Thus we let

$$
\mathrm{ER}^{F} \equiv \mathrm{E}\left[\left(\tilde{p}^{F}-\tilde{v}\right) \tilde{x}\right]=-\bar{x} \mathrm{E}\left(\tilde{v}-\tilde{p}^{F}\right)-\lambda^{F} \operatorname{var}(\tilde{x}),
$$

where the second equality follows from the price function in equation (1) and immediately implies that the expected revenue of liquidity traders decreases in the cost of capital and increases in market liquidity. By default, in this economy the exchange has a profit of $\pi^{F}=0$.

\section{B. ECONOMY ${ }^{D}$ versus ECONOMYF}

In $\mathrm{ECONOMY}^{\mathrm{D}}$ we denote the equilibrium price of the risky asset resulting from the exchange's optimal price for information, $q^{*}$, by $\tilde{p}^{*}$. This price is obtained by setting $\mu=\mu^{*}$ in Proposition 1 . The profit-maximizing exchange charges a price $q^{*}$ so that each trader is indifferent between becoming price informed and paying $q^{*}$ and remaining price uninformed. Thus, the welfare of each rational trader is the certainty equivalent of the ex ante utility of a priceuninformed trader:

$$
\mathrm{CE}^{D}=-\frac{1}{\gamma} \ln \left(-\mathrm{E}\left[\mathrm{VU}\left(\tilde{s}_{i}\right)\right]\right)
$$

where

$$
\mathrm{E}\left[\mathrm{VU}\left(\tilde{s}_{i}\right)\right]=-\sqrt{\frac{\operatorname{var}\left(\tilde{v}-\tilde{p}^{*} \mid \tilde{s}_{i}\right)}{\operatorname{var}\left(\tilde{v}-\tilde{p}^{*}\right)}} \exp \left\{-\frac{\left[\mathrm{E}\left(\tilde{v}-\tilde{p}^{*}\right)\right]^{2}}{2 \operatorname{var}\left(\tilde{v}-\tilde{p}^{*}\right)}\right\} .
$$

In this economy the expected revenue of liquidity traders is:

$$
\mathrm{ER}^{D} \equiv \mathrm{E}\left[\left(\tilde{p}^{*}-\tilde{v}\right) \tilde{x}\right]=-\bar{x} \mathrm{E}\left(\tilde{v}-\tilde{p}^{*}\right)-\lambda^{*} \operatorname{var}(\tilde{x})
$$

and the exchange has a profit of $\pi^{D}=\mu^{*} B\left(\mu^{*}\right)$. Combining Corollaries 1-4 and using the expressions of $\mathrm{ER}^{F}$ and $\mathrm{ER}^{D}$, we have the following proposition:

\footnotetext{
${ }^{12}$ The microstructure literature has a long tradition of using the expected trading cost to represent the welfare of noise traders (e.g., Chowdhry and Nanda (1991), Subrahmanyam (1991), Leland (1992), and Foucault and Gehrig (2008)).
} 
Proposition 3. Price informativeness is lower, the cost of capital and return volatility are higher, liquidity is lower, and liquidity traders have a lower expected revenue in $\mathrm{ECONOMY}^{\mathrm{D}}$ than in $\mathrm{ECONOMY}^{\mathrm{F}}$.

Thus, allowing the exchange to sell price information at the profitmaximizing price reduces price informativeness and increases volatility. It also lowers liquidity and lowers liquidity traders' expected revenue. For rational traders, the welfare effect of allowing the exchange to sell access to price information is ambiguous because of two competing effects: i) ECONOMY ${ }^{\mathrm{D}}$ is less transparent, and rational traders can make more profit from trading with liquidity traders; and ii) in ECONOMY ${ }^{\mathrm{D}}$ rational traders have to pay a cost of $q^{*}$ to purchase the price data, whereas it is free in $\mathrm{ECONOMY}^{\mathrm{F}}$.

To ask which economy (ECONOMY ${ }^{\mathrm{F}}$ or ECONOMY ${ }^{\mathrm{D}}$ ) is better, we need to specify an evaluation criterion. There are two possible approaches to defining such a criterion, one positive and one normative. The positive approach is to examine the behavior of positive variables that represent market quality. Those variables include market efficiency (price discovery), return volatility, liquidity, and the cost of capital, which are central to the current regulatory debates. ${ }^{13}$

The normative approach is to examine the payoffs of each group of players in the economy, that is, CE for rational traders, ER for liquidity traders, and $\pi$ for the exchange. This approach allows us to discuss who wins and who loses under a certain policy. Whether the outcomes are desirable depends on the regulation goal. In our model, liquidity traders can be interpreted as uninformed small retail traders, and rational traders are more in line with speculators, such as institutional traders. If the regulator wishes to protect small traders, then the welfare ER of liquidity traders is the relevant measure. If the regulator wishes to maximize total surplus, then the sum $(\mathrm{CE}+\mathrm{ER}+\pi)$ across all participants may be a relevant measure for social welfare. ${ }^{14}$

Table 2 illustrates the comparison between ECONOMY ${ }^{\mathrm{D}}$ and ECONOMY ${ }^{\mathrm{F}}$, both from a positive perspective and from a normative perspective, for the parameter configuration given by Table 1 . Consistent with Proposition 3, we see that market efficiency and liquidity decrease and the cost of capital and return volatility

\footnotetext{
${ }^{13}$ Both regulators and academia use these positive variables to capture market quality. In reviewing the market data issues, the Committee of European Securities Regulators (CESR) expressed its concern about what role price information plays "in achieving efficient price discovery and facilitating the achievement and monitoring of best execution" (CESR Technical Advice (July 2010)). The recent debate on dark pools also points to price discovery, return volatility, and liquidity as the representative market quality variables. For example, the European Commission remarks that the "increased use of dark pools . . raise[s] regulatory concerns as it may ultimately affect the quality of the price discovery mechanism on the 'lit' markets" (Review of the Markets in Financial Instruments Directive (MiFID), 2010). Thomas Callahan, an NYSE Euronext executive vice president, commented that alternative trading systems "can harm price discovery and worsen short-term volatility" ("NYSE Euronext Asks Congress to Press the SEC On Dark Pools," Trader's Magazine (June 10, 2009)). Proponents of the recent short-sales bans in 2008 also cite lowering volatility as one justification for such restrictions. Many academic papers use these positive variables to study the implications of regulations on various issues (e.g., Boehmer, Jones, and Zhang (2013) on short-sales ban; Wang (2016) on margin requirement; Zhu (2014) on dark pools).

${ }^{14}$ Of course, the regulator could also consider some weighted sum of nonlinear transformations of these variables. We determine the individual variables, and the reader can construct any desired transformation from them.
} 
TABLE 2

Implications of Selling Price Information in Economies with Exogenous Fundamental Information

\begin{tabular}{|c|c|c|}
\hline Variables & ECONOMYD & ECONOMYF \\
\hline \multicolumn{3}{|l|}{ Positive Implications } \\
\hline Fraction of price-informed traders $(\mu)$ & 0.1383 & 1.00 \\
\hline Price informativeness $(m)$ & 0.4313 & 1.6667 \\
\hline Cost of capital $(E(\tilde{V}-\tilde{p}))$ & 0.3113 & 0.0730 \\
\hline Return volatility $(\sigma(\tilde{v}-\tilde{p}))$ & 0.1795 & 0.1692 \\
\hline Liquidity $(1 / \lambda)$ & 1.3129 & 4.2529 \\
\hline \multicolumn{3}{|l|}{ Normative Implications } \\
\hline Rational traders (CE) & 0.1022 & 0.0581 \\
\hline Liquidity traders (ER) & -0.5017 & -0.1318 \\
\hline Exchange profit $(\pi)$ & 0.0367 & 0.00 \\
\hline Total $(\mathrm{CE}+\mathrm{ER}+\pi)$ & -0.3628 & -0.0737 \\
\hline
\end{tabular}

increase if the exchange is allowed to sell price information. So, from a positive perspective, market quality is lower in ECONOMY ${ }^{\mathrm{D}}$ than in ECONOMY ${ }^{\mathrm{F}}$. In terms of normative implications, by Proposition 3, liquidity traders are worse off in ECONOMY ${ }^{\mathrm{D}}$ than in ECONOMY ${ }^{\mathrm{F}}$. By construction, the exchange is better off in $\mathrm{ECONOMY}^{\mathrm{D}}$ because in $\mathrm{ECONOMY}^{\mathrm{F}}$ it has a profit of 0 . In addition, for this numerical example, rational traders are better off in $\mathrm{ECONOMY}^{\mathrm{D}}$, because the aforementioned positive effect dominates. In terms of aggregate welfare $(\mathrm{CE}+\mathrm{ER}+\pi)$, the society is worse off in $\mathrm{ECONOMY}^{\mathrm{D}}$ than in ECONOMY ${ }^{\mathrm{F}}$.

Note that although the exchange extracts profits from traders as a monopolist, the interactions among the exchange and traders are not a zero-sum game. This is because not all market participants are risk neutral. Rational traders are risk averse, and we measure their welfare with a certainty equivalent. However, we effectively treat liquidity traders as risk neutral when we measure their welfare with a expected revenue. So, the trading benefit accruing to rational traders is generally smaller than the trading loss borne by liquidity traders. This implies a negative aggregate welfare. ${ }^{15}$

This negative aggregate welfare may appear odd at first glance. We interpret it in two ways, both of which are related to liquidity traders. First, note that the welfare measure ER for liquidity traders has ignored their liquidity/hedging needs decided outside the financial markets. So, if we instead incorporated these unmodeled benefits into our computation, we would expect that the aggregate welfare measure would turn positive, because otherwise liquidity traders would not have participated in trading. Second, we may literally interpret liquidity traders as noise traders who trade too excessively based on their wrong beliefs, as highlighted by the behavioral finance literature (e.g., Odean (1999), Barber and Odean (2001)). So, the negative number of ER is a measure of trading loss of noise traders, and this can result in negative aggregate welfare.

\footnotetext{
${ }^{15}$ Only changes in welfare matter for evaluating alternative regulations, so the actual values of welfare are not important for our analysis.
} 


\section{Endogenous Information Acquisition}

We now allow rational traders to decide whether to acquire fundamental information in addition to deciding whether to acquire price information. That is, traders can still purchase price data $\tilde{p}$ from the exchange at price $q$, but they are no longer endowed with signals $\tilde{s}_{i}$. Instead, if rational trader $i$ wants to observe signals $\tilde{s}_{i}$, the trader has to pay a cost $c>0$. The baseline model analyzed in previous sections corresponds to the special case of $c=0$. As in Admati and Pfleiderer (1987), this cost $c$ admits two interpretations. First, there may be an information production technology available to each individual, and $c$ is the cost that a trader spends to produce the information. For example, interpreting the voluminous information released by companies requires skill and effort, and $c$ represents the cost of acquiring these skills and expending this effort. Second, there may be an information vendor who produces information and then sells it to investors. A good example is a brokerage analyst who sells various reports and newsletters to clients. In this section, we are agnostic about these interpretations and take $c$ as exogenous. In next section, we endogenize $c$ under the second interpretation. Of course, the price $q$ of price data is still endogenously derived from the profitmaximizing behavior of the exchange.

\section{A. The Equilibrium at the Trading Stage}

With two information purchase decisions there are potentially four trader types: "PS-informed" (of mass $\mu_{p s}$, who purchase both $\tilde{p}$ and $\tilde{s}_{i}$ ), "S-informed" (of mass $\mu_{s}$, who purchase $\tilde{s}_{i}$ ), "P-informed" (of mass $\mu_{p}$, who purchase $\tilde{p}$ ), and "Uninformed" (of mass $\mu_{u}$, without any information). At the trading stage, there is an REE in which the price function has the form given by equation (1), and observing price is equivalent to observing the signal $\tilde{s}_{p}$, given by equation (2). Using computations similar to those in the previous sections, it is easy to show that the demand functions of the four types of traders are, respectively,

$$
\begin{aligned}
D_{p s}\left(\tilde{p} ; \tilde{s}_{i}\right) & =\frac{\mathrm{E}\left(\tilde{v} \mid \tilde{p}, \tilde{s}_{i}\right)-\tilde{p}}{\gamma \operatorname{var}\left(\tilde{v} \mid \tilde{p}, \tilde{s}_{i}\right)}, \\
D_{s}\left(\tilde{s}_{i}\right) & =\frac{\mathrm{E}\left(\tilde{v}-\tilde{p} \mid \tilde{s}_{i}\right)}{\gamma \operatorname{var}\left(\tilde{v}-\tilde{p} \mid \tilde{s}_{i}\right)}, \\
D_{p}(\tilde{p}) & =\frac{\mathrm{E}(\tilde{v} \mid \tilde{p})-\tilde{p}}{\gamma \operatorname{var}(\tilde{v} \mid \tilde{p})}, \quad \text { and } \\
D_{u} & =\frac{\mathrm{E}(\tilde{v}-\tilde{p})}{\gamma \operatorname{var}(\tilde{v}-\tilde{p})} .
\end{aligned}
$$

The market-clearing condition is

$$
\int_{0}^{\mu_{p s}} D_{p s}\left(\tilde{p} ; \tilde{s}_{i}\right) d i+\int_{0}^{\mu_{s}} D_{s}\left(\tilde{s}_{i}\right) d i+\mu_{p} D_{p}(\tilde{p})+\mu_{u} D_{u}=\tilde{x} .
$$

Solving the market-clearing condition for $\tilde{p}$ yields the following proposition, which generalizes Proposition 1 to an economy with four types of traders: 
Proposition 4. In the economy with four types of traders, there exists a partially revealing REE, in which the price function is

$$
\tilde{p}=\alpha+\beta \tilde{v}-\lambda \tilde{x},
$$

where

$$
\begin{aligned}
& \beta=\frac{\gamma m+\left(\mu_{p s}+\mu_{p}\right) m^{2} \rho_{x}}{\mu_{p s} \rho_{\varepsilon}+\left(\mu_{p s}+\mu_{p}\right)\left(\rho_{v}+m^{2} \rho_{x}\right)}, \\
& \lambda=\frac{\gamma+\left(\mu_{p s}+\mu_{p}\right) m \rho_{x}}{\mu_{p s} \rho_{\varepsilon}+\left(\mu_{p s}+\mu_{p}\right)\left(\rho_{v}+m^{2} \rho_{x}\right)}, \\
& \alpha=(1-\beta) \bar{v}+\lambda \bar{x} \\
& -\frac{\gamma \bar{x}}{\left[\begin{array}{c}
\mu_{p s} \operatorname{var}^{-1}\left(\tilde{v}-\tilde{p} \mid \tilde{p}, \tilde{s}_{i}\right)+\mu_{p} \operatorname{var}^{-1}(\tilde{v}-\tilde{p} \mid \tilde{p}) \\
+\mu_{s} \operatorname{var}^{-1}\left(\tilde{v}-\tilde{p} \mid \tilde{s}_{i}\right)+\mu_{u} \operatorname{var}^{-1}(\tilde{v}-\tilde{p})
\end{array}\right]},
\end{aligned}
$$

where $m$ is determined by

$$
L(m)=0
$$

with

$$
\begin{aligned}
L(m) \equiv & \mu_{p s}+\mu_{s} \\
& \times \frac{\left[\mu_{p s} \rho_{\varepsilon}+\left(\mu_{p s}+\mu_{p}\right) \rho_{v}-\gamma m\right]\left[\mu_{p s} \rho_{\varepsilon}+\left(\mu_{p s}+\mu_{p}\right)\left(\rho_{v}+m^{2} \rho_{x}\right)\right]}{\left[\mu_{p s} \rho_{\varepsilon}+\left(\mu_{p s}+\mu_{p}\right) \rho_{v}-\gamma m\right]^{2}+\left[\gamma+\left(\mu_{p s}+\mu_{p}\right) m \rho_{x}\right]^{2} \frac{\rho_{v}+\rho_{\varepsilon}}{\rho_{x}}} \\
& -\frac{\gamma m}{\rho_{\varepsilon}},
\end{aligned}
$$

and where

$$
\begin{aligned}
\operatorname{var}\left(\tilde{v}-\tilde{p} \mid \tilde{p}, \tilde{s}_{i}\right) & =\frac{1}{\rho_{v}+\rho_{\varepsilon}+m^{2} \rho_{x}}, \\
\operatorname{var}(\tilde{v}-\tilde{p} \mid \tilde{p}) & =\frac{1}{\rho_{v}+m^{2} \rho_{x}}, \\
\operatorname{var}\left(\tilde{v}-\tilde{p} \mid \tilde{s}_{i}\right) & =(1-\beta)^{2} \frac{1}{\rho_{v}+\rho_{\varepsilon}}+\lambda^{2} \frac{1}{\rho_{x}}, \\
\operatorname{var}(\tilde{v}-\tilde{p}) & =(1-\beta)^{2} \frac{1}{\rho_{v}}+\lambda^{2} \frac{1}{\rho_{x}} .
\end{aligned}
$$

\section{B. The Equilibrium at the Information Acquisition Stage}

We next characterize how traders purchase information, which in turn determines the demand function faced by the exchange. To do so, we first compute how a trader values the to-be-purchased information ex ante. Then, based on the expressions of information values, we construct eight possible cases of information allocations that are consistent with an equilibrium (i.e., the "viable allocations" in Admati and Pfleiderer (1987)). Finally, we analytically characterize these eight cases in terms of the information price space $(q, c)$. We show that for any given $(q, c) \in \mathbb{R}_{++}^{2}$, there exists a unique equilibrium trader distribution $\left(\mu_{p s}, \mu_{p}, \mu_{s}, \mu_{u}\right)$. 


\section{The Value of Information}

We follow Admati and Pfleiderer ((1987), p. 85) and define the "value of information" as the maximum amount (in terms of foregone consumption) a trader would pay for the opportunity to see the information before trading. That is, the information value is the difference between the certainty equivalent of a trader with the information and that of a trader without it. So, the value of the information $\tilde{p}$ would be the difference between the certainty equivalent of a P-informed trader and that of an uninformed trader. We can define the value of observing the signal $\tilde{s}_{i}$ and of observing the two signals $\left\{\tilde{p}, \tilde{s}_{i}\right\}$ similarly.

Using the expressions of the demand function and the moment-generating function of $\chi^{2}$ distributions, we can compute the ex ante utilities of PS-informed, $\mathrm{S}$-informed, P-informed, and uninformed traders are as follows:

$$
\begin{aligned}
\mathrm{E}\left[V_{p s}\left(\tilde{p}, \tilde{s}_{i}\right)\right] & =\sqrt{\frac{\operatorname{var}\left(\tilde{v}-\tilde{p} \mid p, \tilde{s}_{i}\right)}{\operatorname{var}(\tilde{v}-\tilde{p})}} e^{\gamma(c+q)} \mathrm{E}\left[V_{u}\right], \\
\mathrm{E}\left[V_{p}(\tilde{p})\right] & =\sqrt{\frac{\operatorname{var}(\tilde{v}-\tilde{p} \mid \tilde{p})}{\operatorname{var}(\tilde{v}-\tilde{p})}} e^{\gamma q} \mathrm{E}\left[V_{u}\right], \\
\mathrm{E}\left[V_{s}\left(\tilde{s}_{i}\right)\right] & =\sqrt{\frac{\operatorname{var}\left(\tilde{v}-\tilde{p} \mid \tilde{s}_{i}\right)}{\operatorname{var}(\tilde{v}-\tilde{p})}} e^{\gamma c} \mathrm{E}\left[V_{u}\right], \quad \text { and } \\
\mathrm{E}\left[V_{u}\right] & =-\exp \left\{-\frac{[\mathrm{E}(\tilde{v}-\tilde{p})]^{2}}{2 \operatorname{var}(\tilde{v}-\tilde{p})}\right\} .
\end{aligned}
$$

Accordingly, their certainty equivalents are, respectively, $-\gamma^{-1} \ln \left(-\mathrm{E}\left[V_{p s}\left(\tilde{p}, \tilde{s}_{i}\right)\right]\right)$, $-\gamma^{-1} \ln \left(-\mathrm{E}\left[V_{p}(\tilde{p})\right]\right),-\gamma^{-1} \ln \left(-\mathrm{E}\left[V_{s}\left(\tilde{s}_{i}\right)\right]\right)$ and $-\gamma^{-1} \ln \left(-\mathrm{E}\left[V_{u}\right]\right)$.

Let $\phi_{p s}, \phi_{p}$, and $\phi_{s}$ denote the value of information $\left\{\tilde{p}, \tilde{s}_{i}\right\},\{\tilde{p}\}$, and $\left\{\tilde{s}_{i}\right\}$, respectively. So, we have:

$$
\begin{aligned}
\phi_{p s} & \equiv-\frac{1}{\gamma} \ln \left(-\mathrm{E}\left[V_{p s}\left(\tilde{p}, \tilde{s}_{i}\right)\right]\right)-\left(-\frac{1}{\gamma} \ln \left(-\mathrm{E}\left[V_{u}\right]\right)\right) \\
& =\frac{1}{2 \gamma} \ln \left[\frac{\operatorname{var}(\tilde{v}-\tilde{p})}{\operatorname{var}\left(\tilde{v}-\tilde{p} \mid p, \tilde{s}_{i}\right)}\right], \\
\phi_{p} & \equiv-\frac{1}{\gamma} \ln \left(-\mathrm{E}\left[V_{p}(\tilde{p})\right]\right)-\left(-\frac{1}{\gamma} \ln \left(-\mathrm{E}\left[V_{u}\right]\right)\right) \\
& =\frac{1}{2 \gamma} \ln \left[\frac{\operatorname{var}(\tilde{v}-\tilde{p})}{\operatorname{var}(\tilde{v}-\tilde{p} \mid \tilde{p})}\right], \\
\phi_{s} & \equiv-\frac{1}{\gamma} \ln \left(-\mathrm{E}\left[V_{s}\left(\tilde{s}_{i}\right)\right]\right)-\left(-\frac{1}{\gamma} \ln \left(-\mathrm{E}\left[V_{u}\right]\right)\right) \\
& =\frac{1}{2 \gamma} \ln \left[\frac{\operatorname{var}(\tilde{v}-\tilde{p})}{\operatorname{var}\left(\tilde{v}-\tilde{p} \mid \tilde{s}_{i}\right)}\right] .
\end{aligned}
$$

\section{Learning Complementarities and Viable Information Allocations}

Equipped with the notion of information value, we can also examine whether the two pieces of information $\tilde{p}$ and $\tilde{s}_{i}$ are complementary, which yields insight 
into whether a trader is more likely to purchase the two signals simultaneously than separately, thereby restricting the possible equilibrium information allocations. In a similar spirit as Admati and Pfleiderer ((1987), p. 87), the two signals $\tilde{p}$ and $\tilde{s}_{i}$ are complements if and only if $\phi_{p s} \geq \phi_{p}+\phi_{s}$, and the two signals $\tilde{p}$ and $\tilde{s}_{i}$ are substitutes if and only if $\phi_{p s} \leq \phi_{p}+\phi_{s}$. The two pieces of information $\tilde{p}$ and $\tilde{s}_{i}$ are complementary in our economy, as formalized in the following proposition:

Proposition 5. Learning Complementarities. In any equilibrium allocation of information, we have $\phi_{p s}>\phi_{p}+\phi_{s}$, and thus the two signals $\tilde{p}$ and $\tilde{s}_{i}$ are complements.

Thus, the extra benefit of observing price information $\tilde{p}$ is higher for S-informed traders than for uninformed traders. Similarly, the benefit of observing fundamental information $\tilde{s}_{i}$ is higher for P-informed traders than for uninformed traders. ${ }^{16}$ This result may appear to be counterintuitive because Admati and Pfleiderer (1986) show that the possibility for traders to extract information from prices reduces the fee they are willing to pay for private fundamental information, suggesting that price information and fundamental information are substitutes rather than complements. This argument would be correct if traders need to forecast only the asset's future value $\tilde{v}$ when they trade the asset, as is the case in Admati and Pfleiderer (1986), where traders can freely see the price, because the trading on fundamental information causes the price to be very informative about $\tilde{v}$ as well. However, in our economy, traders do not observe the price, and so they need to forecast $(\tilde{v}-\tilde{p})$ rather than just $\tilde{v}$. In this case, knowing both $\tilde{p}$ and $\tilde{s}_{i}$ is particularly useful because knowledge of $\tilde{p}$ completely removes the price risk, and at the same time, both $\tilde{p}$ and $\tilde{s}_{i}$ help to pin down the fundamental value $\tilde{v} .^{17}$

Proposition 5 shows that P-informed and S-informed traders cannot coexist. Intuitively, because of the complementarity of the two signals, traders have less incentive to acquire them individually rather than jointly. We state this result in the following corollary:

Corollary 5. P- and S-Informed Traders Cannot Coexist. In any information market equilibrium, it is not possible to have both $\mathrm{P}$-informed and $\mathrm{S}$-informed active traders. That is, in any equilibrium, at least one of the two masses $\mu_{p}$ and $\mu_{s}$ must be 0 .

In Proposition 4, $m$ is implicitly determined by equations (20) and (21). Corollary 5 allows us to analytically solve for $m$, because when $\mu_{s}$ or $\mu_{p}$ is 0 , equations (20) and (21) admit an analytical solution.

\footnotetext{
${ }^{16}$ Boulatov and Dierker (2007) provide a related result showing that price data are more valuable to those traders with more precise private fundamental information. Our analysis differs from theirs in two important ways. First, the precision of price information in their model is exogenous. They employ a standard Kyle (1989) model with risk-neutral traders and market makers. The price is therefore set by market makers who receive an exogenous new piece of dividend information, which is incorporated into prices that will be sold by the exchange. Second, they do not allow traders to acquire fundamental information endogenously. We believe these two issues are essential features of price information because they are responsible for the feedback effects that are unique to price information.

${ }^{17}$ Note that in our model, purchasing price information means both i) purchasing the right to submit price-contingent orders and ii) purchasing a signal on the asset value. The first feature is important for the complementarity result in Proposition 5. See related discussions in Section VI.B.
} 
Corollary 6. (i) When $\mu_{s}=0$, we have $m=\gamma^{-1} \mu_{p s} \rho_{\varepsilon}$. (ii) When $\mu_{p}=0$, we have

$$
m=\frac{\mu_{p s} \rho_{\varepsilon}\left(\gamma^{2}+\rho_{v} \rho_{x} \mu_{p s}^{2}+\rho_{x} \rho_{\varepsilon} \mu_{p s}^{2}+\mu_{s} \rho_{v} \rho_{x} \mu_{p s}+\mu_{s} \rho_{x} \rho_{\varepsilon} \mu_{p s}\right)}{\gamma\left(\gamma^{2}+\rho_{v} \rho_{x} \mu_{p s}^{2}+\rho_{x} \rho_{\varepsilon} \mu_{p s}^{2}+\mu_{s} \rho_{x} \rho_{\varepsilon} \mu_{p s}\right)}
$$

and, moreover, $\partial m / \partial \mu_{p s}>0$ and $\partial m / \partial \mu_{s}>0$.

The fact that $\mu_{p}$ and $\mu_{s}$ cannot both be positive in Corollary 5 puts restrictions on the possible information allocations in equilibrium. In addition, in order to maintain a positive demand for price data and hence nonzero profit for the exchange, at least one of the two masses $\mu_{p s}$ and $\mu_{p}$ must be positive in equilibrium, which further limits the possible allocations consistent with equilibrium. Overall, we have eight cases of viable information allocation, as illustrated in Table 3.

TABLE 3

Supported Trader-Type Allocations

Table 3 presents various cases of trader-type allocations together with the equilibrium conditions characterizing these respective cases.

Cases

(1) $\mu_{p s}>0, \mu_{p}=0, \mu_{s}>0, \mu_{u}>0$
(2) $\mu_{p s}>0, \mu_{p}=0, \mu_{s}>0, \mu_{u}=0$
(3) $\mu_{p s}>0, \mu_{p}>0, \mu_{s}=0, \mu_{u}>0$
(4) $\mu_{p s}>0, \mu_{p}>0, \mu_{s}=0, \mu_{u}=0$
(5) $\mu_{p s}>0, \mu_{p}=0, \mu_{s}=0, \mu_{u}=0$
(6) $\mu_{p s}=1$
(7) $\mu_{p s}=0, \mu_{p}>0, \mu_{s}=0, \mu_{u}>0$
(8) $\mu_{p}=1$
Equilibrium Conditions

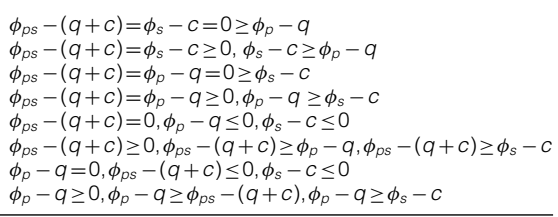

\section{The Existence and Uniqueness of Equilibrium}

In Table 3, for each possible case of information allocation, we present the equilibrium conditions in terms of information value and information cost. Specifically, for a trader-type distribution $\left(\mu_{p s}, \mu_{p}, \mu_{s}, \mu_{u}\right) \in \mathbb{R}_{+}^{4}$ to be an equilibrium at the information acquisition stage, we require the following two "no-deviation" conditions to be satisfied: (i) the active types of traders (i.e., those types of traders with positive $\mu \mathrm{s}$ ) must have the same utility level, and (ii) the inactive types of traders (i.e., those types of traders with $\mu=0$ ), cannot have higher utility levels than the active types. Condition (i) is required, because if some active type, say, type $a$, has a lower utility level than another active type, say, type $b$, then type $a$ traders would like to switch to become type $b$ traders, and so type $a$ will no longer be an active type. Condition (ii) is required for a similar reason.

We then further characterize these equilibrium conditions in terms of information prices $(q, c)$ and other primitive parameters. In particular, we show that the sets of $(q, c)$ supporting the eight cases of information allocation jointly cover $\mathbb{R}_{++}^{2}$. This implies that for any given information price pair $(q, c)$, we can always find an equilibrium at the information acquisition stage. In addition, we can analytically characterize this equilibrium and show that it is unique. The details are provided in 8 lemmas in the Internet Appendix (available at www.jfqa.org), and here we present only the following proposition on the existence and uniqueness of equilibrium. 
Proposition 6. For any $c>0$ and $q>0$, there exists a unique equilibrium $\left(\mu_{p s}, \mu_{p}, \mu_{s}, \mu_{u}\right)$ at the information acquisition stage.

Figure 3 plots the eight cases of supported trader-type distributions in the space of $(q, c)$ for the parameter values given in Table 1. Generally speaking, as $q$ becomes higher, fewer traders will purchase price data $\tilde{p}$, and similarly, as $c$ becomes higher, fewer traders will acquire the fundamental information $\tilde{s}_{i}$.

The three graphs in Figure 3 differ only in the upper boundary of the Case (1) information allocations in which PS-informed, S-informed, and uninformed traders are active. Specifically, in Graph C, we have $\gamma \geq \sqrt{\rho_{\varepsilon} \rho_{x}}$, and the upper boundary of Case (1) decreases with $q$, whereas in Graphs A and B, the upper boundary of Case (1) first increases and then decreases with $q$. Graphs A and $\mathrm{B}$ are further differentiated by whether the maximum value of $c$ in Case (1) exceeds a threshold cost of fundamental information, $\underline{c}=(2 \gamma)^{-1} \ln \left(1+\rho_{\varepsilon}\left(\rho_{v}+\right.\right.$ $\left.\left.\left(\rho_{\varepsilon} / \gamma\right)^{2} \rho_{x}\right)^{-1}\right)$, as defined by equation (31) in ECONOMY ${ }^{\mathrm{F}}$, which we will discuss shortly. In Graph A, we have $\gamma<0.5 \sqrt{\left(\rho_{v} \rho_{\varepsilon} \rho_{x}\right) /\left(\rho_{v}+\rho_{\varepsilon}\right)}$, and the maximum value of $c$ in Case (1) is greater than $\underline{c}$, whereas in Graph $\mathrm{B}$, we have $0.5 \sqrt{\left(\rho_{v} \rho_{\varepsilon} \rho_{x}\right) /\left(\rho_{v}+\rho_{\varepsilon}\right)} \leq \gamma<\sqrt{\rho_{\varepsilon} \rho_{x}}$, and the maximum value of $c$ in Case (1) does not exceed $\underline{c}$.

FIGURE 3

Trader Distributions with Endogenous Fundamental Information

Figure 3 plots the regimes of trader types in equilibrium in the space $(q, c)$ when fundamental information is endogenous. The absolute risk-aversion coefficient $\gamma$ is $1.5,3$, and 5, respectively in Graphs A, B, and C. All other parameter values are fixed at the values in Table 1.
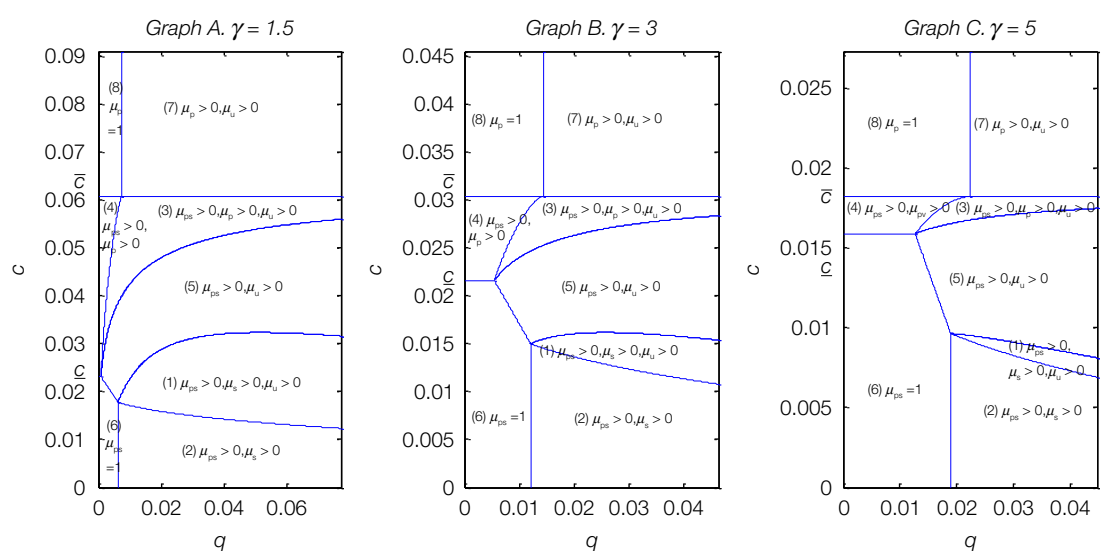

\section{Information Acquisition in ECONOMYF}

We now describe equilibrium information acquisition decisions in the benchmark economy, ECONOMY ${ }^{\mathrm{F}}$, in which all traders observe price information for free (i.e., $q=0$ ). In this economy, traders are either PS-informed or P-informed; that is, $\mu_{p}+\mu_{p s}=1$. Actually, from Figure 3, we can see that as long as the price $q$ is sufficiently low or, more specifically, as long as

$$
q \leq(2 \gamma)^{-1} \ln \left[1+\gamma^{6} \rho_{v}\left(\rho_{x}\left(\gamma^{2} \rho_{v}+\gamma^{2} \rho_{\varepsilon}+\rho_{x} \rho_{\varepsilon}^{2}\right)^{2}\right)^{-1}\right]
$$


the relevant trader distributions are Cases (4), (6), and (8), and only PS- and P-informed traders are active in these cases.

By Corollary 6, we have $m=\gamma^{-1} \mu_{p s} \rho_{\varepsilon}$. Equations (22), (23), (26), and (27) imply that the benefit of observing fundamental information is

$$
B^{F}\left(\mu_{p s}\right) \equiv \phi_{p s}-\phi_{p}=\frac{1}{2 \gamma} \ln \left[1+\frac{\rho_{\varepsilon}}{\rho_{v}+\left(\mu_{p s} \rho_{\varepsilon} / \gamma\right)^{2} \rho_{x}}\right],
$$

which is decreasing in $\mu_{p s}$.

It will be useful to define two extreme values for the benefit of acquiring private signals. This benefit is largest when no one else is informed, $\mu_{p s}=0$, and it is smallest when everyone else is informed, $\mu_{p s}=1$. Specifically, we define

$$
\begin{aligned}
& \bar{c} \equiv B^{F}(0)=\frac{1}{2 \gamma} \ln \left(1+\frac{\rho_{\varepsilon}}{\rho_{v}}\right) \text { and } \\
& \underline{c} \equiv B^{F}(1)=\frac{1}{2 \gamma} \ln \left(1+\frac{\rho_{\varepsilon}}{\rho_{v}+\left(\frac{\rho_{\varepsilon}}{\gamma}\right)^{2} \rho_{x}}\right) .
\end{aligned}
$$

If the cost of acquiring signals is too large, $c \geq \bar{c}$, then the equilibrium fraction $\mu_{p s}^{F}$ of PS-informed traders is 0. Alternatively, if it is sufficiently low, $c \leq \underline{c}$, then $\mu_{p s}^{F}=$ 1. Finally, if $c \in(\underline{c}, \bar{c}), \mu_{p s}^{F}$ is determined by $B^{F}\left(\mu_{p s}\right)=c$, which is characterized by the following proposition:

Proposition 7. Suppose $\underline{c} \leq c \leq \bar{c}$. The equilibrium fraction $\mu_{p s}^{F}$ of PS-informed traders in ECONOMY ${ }^{\mathrm{F}}$ is determined by

$$
\mu_{p s}^{F}=\frac{\gamma}{\rho_{\varepsilon}} \sqrt{\frac{\frac{\rho_{\varepsilon}}{e^{2 \gamma c}-1}-\rho_{v}}{\rho_{x}}},
$$

and the price informativeness is $m^{F}=\gamma^{-1} \mu_{p s}^{F} \rho_{\varepsilon}$.

\section{The Implications of Selling Price Information}

We next examine how the exchange sets the price $q$ to maximize profit in ECONOMY ${ }^{\mathrm{D}}$, and then we analyze the implications of selling price data. In the Internet Appendix, Part (b) of Lemmas 1-8 analytically characterizes $\left(\mu_{p s}, \mu_{p}, \mu_{s}, \mu_{u}\right)$ for a given information price pair $(q, c)$. This in turn determines the demand function for price data, which is the critical input to the exchange's profit-maximization problem. Figure 4 plots the demand function (Graph A) and the profit function (Graph B) of the exchange, assuming that the cost $c$ of acquiring the fundamental information $\tilde{s}_{i}$ is set at 0.0216 . The other parameters are given by Table 1 . The profit-maximizing price $q^{*}$ is 0.2657 under this parameter configuration, and the resulting equilibrium trader distribution is $\mu_{p s}^{*}=0.1295$ and $\mu_{u}^{*}=0.8705$ (i.e., Case (5) in Table 3).

For the same parameter configuration in Figure 4, the corresponding total amount of fundamental information $\mu_{p s}^{F}$ in ECONOMY ${ }^{\mathrm{F}}$ is 0.9976 . So, relative to $\mathrm{ECONOMY}^{\mathrm{F}}$, where price information is free, selling it in $\mathrm{ECONOMY}^{\mathrm{D}}$ reduces the total amount of fundamental information produced by traders. The intuition for this result follows from the learning complementarity result identified in 


\section{FIGURE 4 \\ Demand for Price Data and Exchange Profit in Economies \\ with Endogenous Fundamental Information}

Figure 4 plots the demand for price data and profit of the exchange as a function of the price $q$ of price data $\tilde{p}$ when traders have to spend a cost $c$ to acquire fundamental information $\tilde{s}_{i}$. The parameter $c$ is set at 0.0216 . All other parameter values are fixed at the values in Table 1.
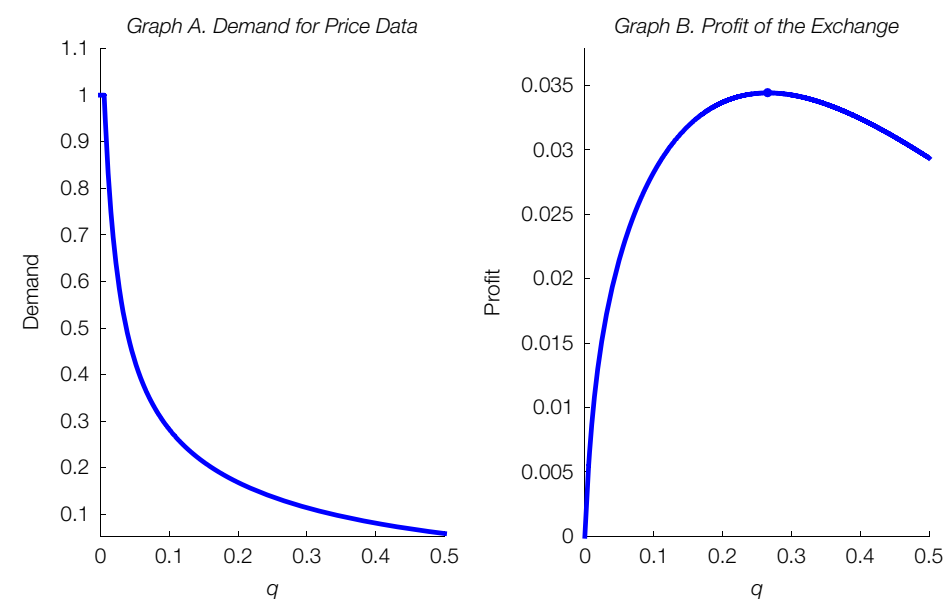

Proposition 5. That is, as $q$ becomes higher, traders have less incentive to purchase price data, and because price information and fundamental information are complementary to each other, they will have less incentive to produce fundamental information as well, other things being equal. Formally, we can prove the following proposition. (The condition $c<\bar{c}$ ensures that there are always some traders acquiring fundamental information. If otherwise $c \geq \bar{c}$, as shown in Figure 3, no traders will produce fundamental information for any value of $q$, and so the total amount of fundamental information is 0 independent of $q$.)

Proposition 8. Crowding-Out Effect. Suppose $c<\bar{c}$. If the optimal price $q^{*}$ set by the exchange is sufficiently high, then there is less fundamental information in ECONOMY $^{\mathrm{D}}$ than in ECONOMY ${ }^{\mathrm{F}}$.

In Table 4, we conduct an exercise similar to Table 2 to illustrate the implications of selling price data in an economy with endogenous fundamental information acquisition. We see that the main message is similar to Table 2. That is, relative to ECONOMY ${ }^{\mathrm{F}}$, all market quality variables become worse in ECONOMY ${ }^{\mathrm{D}}$ : The total amount of information becomes lower, the market becomes less informative and less liquid, and the cost of capital and return volatility become higher. Also, rational traders are better off in $\mathrm{ECONOMY}^{\mathrm{D}}$ than in $\mathrm{ECONOMY}^{\mathrm{F}}$, liquidity traders have lower expected revenue, and aggregate welfare is lower.

Formally, we can show the following proposition:

Proposition 9. ECONOMY ${ }^{\mathrm{D}}$ versus ECONOMY ${ }^{\mathrm{F}}$. Suppose $c \leq c \leq \bar{c}$ and $\gamma \geq$ $0.5 \sqrt{\left(\rho_{v} \rho_{\varepsilon} \rho_{x}\right) /\left(\rho_{v}+\rho_{\varepsilon}\right)}$. Relative to ECONOMYF, ECONOMY ${ }^{\mathrm{D}}$ has a (weakly) lower price informativeness, a higher cost of capital, a higher return volatility, a lower liquidity, and a lower expected revenue of liquidity traders. 
TABLE 4

Implications of Selling Price Information in Economies with Endogenous Fundamental Information

\begin{tabular}{|c|c|c|}
\hline Variables & ECONOMYD & ECONOMYF \\
\hline \multicolumn{3}{|l|}{ Positive Implications } \\
\hline \multicolumn{3}{|l|}{ Trader distributions } \\
\hline PS-informed $\left(\mu_{p s}\right)$ & 0.1295 & 0.9976 \\
\hline P-informed $\left(\mu_{p}\right)$ & 0.00 & 0.0024 \\
\hline S-informed $\left(\mu_{s}\right)$ & 0.00 & 0.00 \\
\hline Uninformed $\left(\mu_{u}\right)$ & 0.8705 & 0.00 \\
\hline Total amount of information $\left(\mu_{p s}+\mu_{s}\right)$ & 0.1295 & 0.9976 \\
\hline Price informativeness $(m)$ & 0.2159 & 1.6627 \\
\hline Cost of capital $(\mathrm{E}(\tilde{V}-\tilde{p}))$ & 0.3490 & 0.0731 \\
\hline Return volatility $(\sigma(\tilde{v}-\tilde{p}))$ & 0.4310 & 0.1693 \\
\hline Liquidity $(1 / \lambda)$ & 1.2564 & 4.2532 \\
\hline \multicolumn{3}{|l|}{ Normative Implications } \\
\hline Rational traders (CE) & 0.1093 & 0.0365 \\
\hline Liquidity traders (ER) & -0.5480 & -0.1319 \\
\hline Exchange profit $(\pi)$ & 0.03442 & 0.00 \\
\hline Total $(\mathrm{CE}+\mathrm{ER}+\pi)$ & -0.4043 & -0.0954 \\
\hline
\end{tabular}

We include the condition $\gamma \geq 0.5 \sqrt{\left(\rho_{v} \rho_{\varepsilon} \rho_{x}\right) /\left(\rho_{v}+\rho_{\varepsilon}\right)}$ in Proposition 9 to exclude the possibility of Graph A of Figure 3. This is because the characterization of the expression of $\mu_{s}$ in Lemma 1 of the Internet Appendix is rather complicated, which precludes a simple analytical proof for the implications of increasing the price $q$ in Case (1) of information allocations. We have used numerical simulations to show that the condition $\gamma \geq 0.5 \sqrt{\left(\rho_{v} \rho_{\varepsilon} \rho_{x}\right) /\left(\rho_{v}+\rho_{\varepsilon}\right)}$ in Proposition 9 is not very stringent. For example, with the parameter configuration in Table 1, we have $0.5 \sqrt{\left(\rho_{v} \rho_{\varepsilon} \rho_{x}\right) /\left(\rho_{v}+\rho_{\varepsilon}\right)}=2.04$, so any $\gamma \geq 2.04$ is sufficient for our result.

\section{Extensions and Discussions}

\section{A. Selling Both Types of Information}

In this subsection, we extend our analysis to endogenize both the price $q$ of price information and the price $c$ of fundamental information. We consider two interesting cases. In both cases, the exchange sells price data, but the seller of fundamental information differs across the two cases. In the first case, an independent information vendor, such as Thomson Reuters, sells (personalized) fundamental information $\tilde{s}_{i}$ to traders. The fundamental information in this case can be understood as the reports and newsletters distributed by analysts, or consumer sentiment data. In the second case, the exchange sells both market and fundamental information. $^{18}$

For a given price pair $(q, c)$, the supported trader distribution $\left(\mu_{p s}, \mu_{p}\right.$, $\mu_{s}, \mu_{u}$ ) is analytically characterized in Part (b) of Lemmas 1-8 provided in

\footnotetext{
${ }^{18} \mathrm{~A}$ recent trend is for exchanges to sell both market and fundamental data. For example, Deutsche Borse sells early access to the Chicago Business Barometer. For discussions of this practice in the press, see "High-Frequency Traders Flat-Out Buying Data ahead of You," Wall Street Journal (Aug. 28, 2012); "High-Frequency Traders Once Again Flat Out Buying Data ahead of You," Wall Street Journal (Sept. 12, 2012); and "Share Trading Volumes Drop after Reuters Changes Release Schedule," Financial Times (July 12, 2013).
} 
the Internet Appendix. This determines the demand $\left(\mu_{p s}+\mu_{p}\right)$ for price information and the demand $\left(\mu_{p s}+\mu_{s}\right)$ for fundamental information. So, the profit $\pi_{\text {price }}$ from selling price data and the profit $\pi_{\mathrm{fdm}}$ from selling fundamental data $\tilde{s}_{i}$ are, respectively,

$$
\pi_{\text {price }}(q, c) \equiv q \times\left(\mu_{p s}+\mu_{p}\right) \quad \text { and } \quad \pi_{\mathrm{fdm}}(q, c)=c \times\left(\mu_{p s}+\mu_{s}\right) .
$$

In the first case, we view the exchange and the information vendor as playing a normal-form game with $q$ and $c$ as their strategies, respectively. That is, each information seller takes the price set by the other seller as given and chooses its own price to maximize its profit. Specifically, their profit maximization problems are, respectively:

$$
\max _{q} \pi_{\text {price }}(q, c) \text { and } \max _{c} \pi_{\mathrm{fdm}}(q, c) .
$$

The first-order conditions of the two problems define the two best response functions, whose intersection in turn determines a Nash equilibrium price vector $\left(q^{*}, c^{*}\right)$. In the second case, the exchange simply chooses $(q, c)$ to maximize the total profit $\pi_{\text {price }}(q, c)+\pi_{\mathrm{fdm}}(q, c)$; that is,

$$
\max _{(q, c)}\left[\pi_{\text {price }}(q, c)+\pi_{\mathrm{fdm}}(q, c)\right] .
$$

The solution $\left(q^{* *}, c^{* *}\right)$ to this problem determines the equilibrium pricing policy of the exchange. In both cases, we can use the computed equilibrium price vector and Lemmas 1-8 of the Internet Appendix to find the corresponding trader distribution and then follow the same steps as in the baseline model to compute the relevant positive and normative variables.

In Figure 5, we graph these two cases for the parameter configuration in Table 1. In Graph A, we assume two different information sellers and plot the two best response functions. We find that they intersect at $q^{*}=0.2350$ and $c^{*}=0.0301$, which is the equilibrium pricing policy in this case. In Graph B, the exchange sells both price and fundamental data. The total profit is maximized at $q^{* *}=0.2090$ and $c^{* *}=0.0042$.

In Table 5, we present the resulting positive and normative variables. In the case with two different information sellers, the trader distribution is $\mu_{p s}^{*}=0.1409$ and $\mu_{u}^{*}=0.8591$, which is comparable to ECONOMY ${ }^{\mathrm{D}}$ in Table 4, where $c$ is fixed at 0.0216 . However, in the second case in which the exchange sells both market and fundamental data, the trader distribution changes to $\mu_{p s}^{*}=0.1731$ and $\mu_{s}^{*}=0.8269$. Note that $\mu_{s}^{*}=0$ in the first case and $\mu_{u}^{*}=0$ in the second case. What is going on here is the following: In the first case, both the exchange and the information vendor are charging a relatively high price, so that if traders purchase information, they would purchase both pieces of information (recall that $\tilde{p}$ and $\tilde{s}_{i}$ are complementary). By contrast, in the second case in which the exchange controls both $q$ and $c$, the exchange finds it more profitable to set a low value of $c$, so that many traders purchase only fundamental information and become S-informed; and the trading of these S-informed traders will in turn make the price very informative, which enhances the exchange's profitability from selling price data.

We find that overall, under the parameter configuration in Table 1, as we go from the first case with two different information sellers to the second case with only one information seller, the price is more informative, the market is 
FIGURE 5

\section{Selling Both Types of Information}

A trader must to pay $q$ to see price information and $c$ to see fundamental information. In Graph A of Figure 5, the exchange sells price information, and an information vendor sells fundamental information. This graph plots the best response functions of the two information sellers. In Graph B, the exchange sells both price information and fundamental information. This graph plots the exchange's overall profit against the information price vector $(q, c)$. The parameter values are fixed at the values in Table 1.
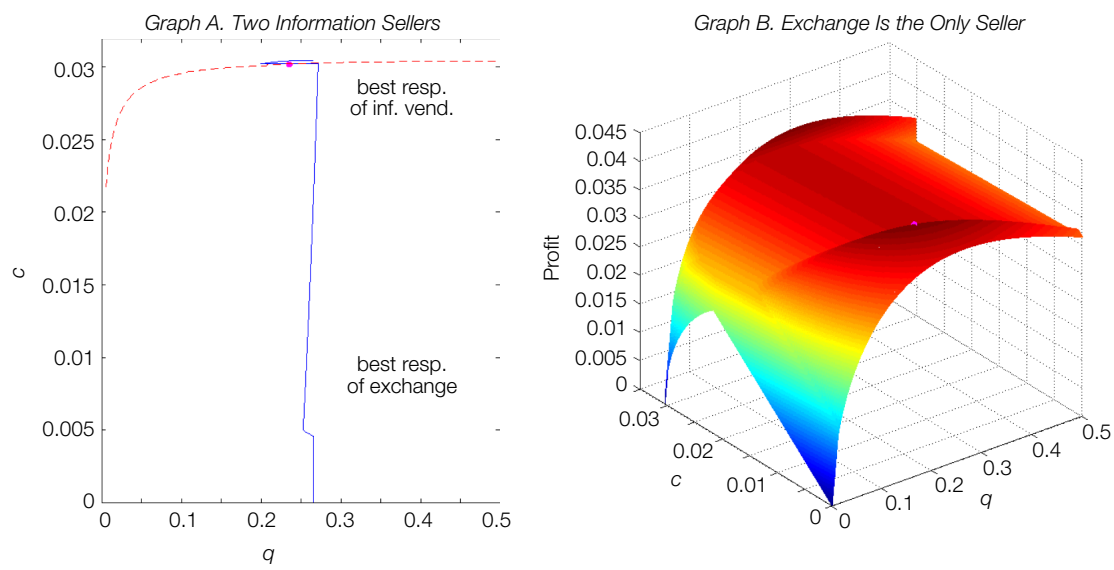

\section{TABLE 5}

\section{Selling Both Types of Information}

A trader needs to pay $q$ to see price information and $c$ to see fundamental information. In one case, the exchange sells both price information and fundamental information. In the other case, the exchange sells price information, and an information vendor sells fundamental information. The parameter values are fixed at the values in Table 1.

\begin{tabular}{l} 
Variables \\
\hline Information Prices \\
Price data $(q)$ \\
Fundamental information $(c)$ \\
Positive Implications \\
Trader distributions \\
PS-informed $\left(\mu_{p s}\right)$ \\
P-informed $\left(\mu_{p}\right)$ \\
S-informed $\left(\mu_{s}\right)$ \\
Uninformed $\left(\mu_{u}\right)$ \\
Total amount of information $\left(\mu_{p s}+\mu_{s}\right)$ \\
Price informativeness $(m)$ \\
Cost of capital $(\mathrm{E}(\tilde{v}-\tilde{p}))$ \\
Return volatility $(\sigma(\tilde{v}-\tilde{p}))$ \\
Liquidity $(1 / \lambda)$ \\
Normative Implications \\
Rational traders $(\mathrm{CE})$ \\
Liquidity traders $(\mathrm{ER})$ \\
Exchange profit \\
Vendor profit \\
Total $\left(\mathrm{CE}+\right.$ ER $\left.+\pi_{\mathrm{price}}+\pi_{\mathrm{fdm}}\right)$ \\
\hline
\end{tabular}

The Exchange Sells Price
Information, and the Vendor

Sells Fundamental Information

\subsection{0}

0.0301

0.1409

0.00

0.00

0.8591

0.1409

0.2349

0.3144

0.4033

1.3595

0.1013

$-0.4983$

0.0331

0.0042

$-0.3597$
The Exchange Sells Both Types of Information

\subsection{0}

0.0042

0.1731

0.00

0.8269

0.00

1.00

0.5555

0.2348

0.3392

1.5970

0.0799

$-0.3914$

0.0403

0.00

$-0.2712$

more liquid, the cost of capital and return volatility are lower, liquidity traders are better off, the exchange earns more profit (than the sum of the two information sellers' profits in the first case), and the aggregate welfare is higher. This all occurs because more traders purchase fundamental information in the second case in which the exchange sells both types of information. 


\section{B. Interpretation of Results}

In this subsection, we discuss some limitations of our modeling approach as well as the interpretation of the results. First, as we noted in Section II, we adopt a 1-period model to capture the multiple feedback effects arising from selling price information. In our model, by construction, price-informed traders differ from price-uninformed traders in two ways: i) price-informed traders see the price, whereas price-uninformed traders do not; and ii) price-informed traders submit demand schedules, whereas price-uninformed traders submit market orders. Demand schedules are close to limit orders in practice, and trading platforms charge distinct fees for limit orders and market orders, which are called maker and taker fees, respectively (Foucault, Kadan, and Kandel (2013)). So, it is interesting to examine separately the roles of i) and ii) in delivering our results.

To do so, we consider the special case in which $\rho_{\varepsilon}$ converges to 0 so that traders' fundamental signals are uninformative. In this limiting case, prices are uninformative about the fundamental $\tilde{v}$ (i.e., $\beta$ and $m$ go to 0 ), and so traders buy price data only for the added flexibility in the specification of their trading plans (i.e., only difference ii) is present). We find that many of our results prior to Section V continue to hold. Specifically, as $q$ decreases and the fraction of price-informed traders, $\mu_{p s}$, increases, the cost of capital and return volatility decrease, liquidity traders become better off, and rational traders become worse off. The price informativeness and liquidity implications are different from Corollaries 1 and 4: Now price informativeness $m$ is not affected by $\mu_{p s}$, and liquidity always increases with $\mu_{p s}$ because only the size effect is active. Because we have shut down the fundamental information, the results in Section $\mathrm{V}$ that are due to interactions between two pieces of information vanish; that is, the learning complementarity result in Proposition 5 and the crowding-out result in Proposition 8 do not arise. ${ }^{19}$

So, to the extent that we interpret the limiting economy with $\rho_{\varepsilon}=0$ as capturing the difference in limit versus market orders, our analysis suggests that the practice of rebating limit orders by platforms promotes market quality. We do not attach much weight to this interpretation of the limiting case of $\rho_{\varepsilon}=0$ because our model does not capture many important features differentiating limit and market orders (e.g., their ability to satisfy the trading needs of traders with different patience parameters as in Colliard and Foucault (2012)). Instead, we put more emphasis on the analysis of the general cases of $\rho_{\varepsilon}>0$, and at the same time we acknowledge that our modeling approach combines the implications of selling price information and allowing traders to use different trading strategies.

We next discuss two important features ignored by our model: that accommodating trading and price discovery are not costless and that, in practice, exchanges are not monopolists. Both features will affect the interpretation of the policy implications of promoting markets with "free" market data.

It is certainly costly for exchanges to provide market data. But once it exists it is a "public good," because use of it by 1 trader does not preclude another trader

\footnotetext{
${ }^{19}$ Actually, the limiting economy of $\rho_{\varepsilon}=0$ can also be viewed as a special case of $c \geq \bar{c}$ in Section $\mathrm{V}$, so that no trader would acquire fundamental information endogenously, and only Cases (7) and (8) of trader distribution are relevant.
} 
from using the same data. ${ }^{20}$ So we can borrow some insights from the public economics literature to study the implications of selling price data. Specifically, suppose that it costs a fixed amount of $K>0$ for the exchange to produce market data and accommodate trading. Because this cost is fixed, the profit maximization behavior of the exchange in ECONOMY ${ }^{\mathrm{D}}$ will not be affected. In this case, simple average cost pricing is a reasonable way (for a regulator) to price the market data produced by a monopoly market (e.g., Brown and Heal (1983)). That is, every market participant could be charged a flat fee, $q^{R}$, for access to the market data where this fee is set so that the exchange recovers its cost. This flat fee could be viewed as a regulated price $q^{R}$ such that the total revenue from selling market data is equal to its fixed cost $K$. In the benchmark ECONOMY ${ }^{\mathrm{F}}$ in our analysis, market data would no longer be free, but instead, they would have a benchmark price $q^{R}$. Because our proofs are all based on comparative statics with respect to $q$, similar results regarding the comparison between ECONOMY ${ }^{\mathrm{D}}$ and ECONOMY ${ }^{\mathrm{F}}$ would continue to hold in this alternative setup as long as $q^{R}<q^{*}$ (see the Appendix).

In our analysis we have assumed a single exchange that fully utilizes its monopoly power in selling market data. In practice, exchanges compete for order flow and trading. It is likely that exchanges subsidize order submission and trading in order to generate the valuable price information that they sell. ${ }^{21}$ If so, it is much less obvious that all traders would be harmed by the sale of price data. Actually, a force of this kind manifests itself in Section VI.A, where the exchange sells both market and fundamental data. There, the exchange sets a very low price $c$ of fundamental data to attract more traders to become $S$-informed in order to generate valuable price data that it can sell. Indeed, the market improves, and liquidity traders are better off relative to an economy where two information vendors sell two types of data independently (see Table 5). However, this economy with the exchange selling both types of information is still worse than the benchmark economy, ECONOMY ${ }^{\mathrm{F}}$ in Table 4, in terms of market quality variables and the aggregate welfare. So, we conjecture that in the presence of multiple exchanges, the competition among them tends to improve market quality and benefit liquidity traders. However, whether this force is strong enough to overturn our analysis pertaining to the monopoly exchange is ambiguous. Conducting a full analysis incorporating competitions among exchanges is beyond the scope of this paper.

\section{Tape Data and Regulatory Policy}

Allowing exchanges to sell price data to traders introduces important price discovery, cost of capital, return volatility, liquidity, and welfare effects. These effects arise because trade information is valuable, both to the traders who know it and to the exchanges that produce it. It is hardly surprising, therefore,

\footnotetext{
${ }^{20}$ Of course, prior use of the data might affect the value of the data to subsequent traders, but that does not make the data a private good.

${ }^{21}$ Whether such subsidization applies to all traders, however, is unclear. In current markets, exchanges have been offering specials benefits (such as co-location, special fee structures and order types) to high-frequency firms that generate massive trading volumes. They have not been subsidizing retail order flow.
} 
that exchanges want to sell trade data and some traders want to buy it. The question of interest, however, is what should regulatory policy be regarding access to and distribution of trading process information?

To understand the context for this debate, consider the current situation in both the United States and Europe with respect to tape data. As noted earlier, the United States has a consolidated tape to which trades and quotes, in principle, must be reported in real time. In practice, some trades (e.g., odd lots) have not been reported to the tape, and the processing of the trades and quotes that are reported requires some time. Whereas in times past these delays ran to several seconds, in 2008 the average latency of the tape was on the order of 20-50 milliseconds, and it is now variously estimated at between 5 and 10 milliseconds. ${ }^{22}$ In Europe, there is no mandatory consolidated tape, and exchanges and trading platforms sell proprietary data feeds. Most U.S. exchanges and markets also provide trade data products, and selling information is a significant source of profit for exchanges worldwide.

The SEC and the CESR are both reviewing their respective market structures with respect to data issues. In Europe, the main question is whether to establish a mandatory consolidated tape. The focus here is on the role price information plays "in achieving efficient price discovery and facilitating the achievement and monitoring of best execution." ${ }^{23}$ In the United States, the issue is proprietary data feeds, with the SEC posing the issue as being one of "fairness." Specifically, the SEC asks "is the existence of any latency, or disparity in information transmitted, fair to investors or other market participants that rely on the consolidated market data feeds and do not use individual trading center data feeds?"24

Both issues essentially involve questions of differential access to price information, and our analysis provides insights into the debate. With respect to the mandatory tape, our analysis shows that market efficiency (i.e., price discovery) and market liquidity are higher and the cost of capital and return volatility are lower when the prices are provided to traders at a lower price. Our analysis also shows that observing price information encourages the production of fundamental information, further improving price discovery. In general, market efficiency is enhanced by greater access to price information, and this is most easily attained with a consolidated tape.

Whether exchanges should also be allowed to sell proprietary data feeds is more complex. Exchanges defend the practice of selling data by noting that such data are not costless to produce, and the revenue they obtain from selling data allows them to invest in the costly trading systems needed to produce highquality trades and quotes. We acknowledge that there is some merit in this defense. Meanwhile, our analysis also suggests that lowering the price of market data can

\footnotetext{
${ }^{22}$ Regulation National Market System (Reg NMS) did not require exchanges to stop providing data feeds, but it did require that they send data to subscribers at the same time that they send the data to the consolidated tape. The current latency difference is thus determined by the time it takes to process the data at the consolidated level. For estimates of current latency speeds, see BATS comment letter (Apr. 10, 2010).

${ }^{23}$ See CESR Technical Advice to the European Commission in the Context of the MiFID Review and Responses to the European Commission Request for Additional Information ((July 2010), p. 28).

${ }^{24}$ See SEC Concept Release on Equity Market Structure ((Apr. 2010), p. 62).
} 
improve market quality in general. Although the exchanges share in the tape revenues from the consolidated tape, selling proprietary data allows trading venues to meet the needs of specialized trading groups. BATS (the third largest trading venue in the United States), for example, actually gives away its data feeds as a competitive inducement to attract high-frequency traders to its trading platform. The exchanges further note that access to the data is "fair" in that they are willing to sell data to any or all traders willing to pay for the data.

We believe that the SEC's query regarding fairness misses the bigger picture. Selling data in our model does result in some traders doing better than others, and it benefits exchanges. Yet, these redistributive effects are only part of the story. ${ }^{25}$ Allowing some traders to purchase better information affects price discovery, the cost of capital, return volatility, market liquidity, information production, and trader welfare. In our view, when evaluating the practice of selling price information, these implications for market quality and trader welfare should be carefully taken into account.

Should the SEC also preclude exchanges from selling data that are not part of the price and quote montage? Here, two issues come to the fore. First, exchanges do sell other market data such as order placements and cancellations, and depth levels away from the quote. Such data can be valuable to traders pursuing particular trading strategies in that the data can help them lower transactions costs. As such, it would seem to be an acceptable practice. A second, and more contentious issue, is whether to allow exchanges to sell fast feeds to fundamental data. In particular, exchanges have begun selling fast access to Fitch ratings changes. These data, which are delivered via the exchanges' high-speed data lines, allows highfrequency traders to learn information before everyone else. Although in isolation this can be viewed as just another way in which traders purchase information, our model suggests that when done in combination with the purchase of fast price data, this practice can have large effects on the market.

From a broader perspective, providing information to some traders but not to all may have unexpected effects, particularly if this practice reduces the transparency of markets to uninformed traders. Easley and O'Hara (2010) demonstrate that ambiguity can reduce participation in markets. To the extent that traders perceive greater ambiguity attaching to markets that selectively sell data, they can opt to trade elsewhere or not at all. ${ }^{26}$ Such an outcome will surely restrict an exchange's data sales even without regulatory involvement.

\footnotetext{
${ }^{25}$ Fairness, per se, is not necessarily an overriding goal for market design because market participants are not all inherently equal. The SEC has not traditionally required a "one size fits all" market structure in recognition of the needs that different traders face.

${ }^{26}$ Such a situation was alleged to have occurred in May 2010 in Europe with respect to data distributed by Chi-X and BATS Europe. In a report from Themis Trading, Arnuk and Saluzzi (2010) alleged that high-speed data packages sold by BATS and NASDAQ-OMX allowed purchasers to discern the existence of hidden orders, thereby disadvantaging hidden limit order traders. Disclosure of this practice prompted European trading platform Turquoise to issue a statement to the effect that its data feeds did not reveal such information. Traders subsequently routed order flow away from Chi-X and BATS, causing a dramatic fall in both venues' trading volume. Chi-X and BATS subsequently changed their data feeds to limit the data revealed.
} 


\section{Appendix. Proofs}

Proof of Corollary 2. Direct computation shows:

$$
\begin{aligned}
& \frac{\partial}{\partial \mu}\left(\frac{\mu}{\operatorname{var}\left(\tilde{v} \mid \tilde{p}, \tilde{s}_{i}\right)}+\frac{1-\mu}{\operatorname{var}\left(\tilde{v}-\tilde{p} \mid \tilde{s}_{i}\right)}\right)=\left[\frac{1}{\operatorname{var}\left(\tilde{v} \mid \tilde{p}, \tilde{s}_{i}\right)}-\frac{1}{\operatorname{var}\left(\tilde{v}-\tilde{p} \mid \tilde{s}_{i}\right)}\right] \\
& \quad+\left[\mu \frac{\partial}{\partial \mu} \frac{1}{\operatorname{var}\left(\tilde{v} \mid \tilde{p}, \tilde{s}_{i}\right)}+(1-\mu) \frac{\partial}{\partial \mu} \frac{1}{\operatorname{var}\left(\tilde{v}-\tilde{p} \mid \tilde{s}_{i}\right)}\right] .
\end{aligned}
$$

We next establish that both bracketed terms are positive, which is a sufficient condition for $\partial C C / \partial \mu<0$ by equation (15).

Clearly, $\operatorname{var}^{-1}\left(\tilde{v}-\tilde{p} \mid \tilde{s}_{i}\right)<\operatorname{var}^{-1}\left(\tilde{v} \mid \tilde{p}, \tilde{s}_{i}\right)$, and as a result, $\operatorname{var}^{-1}\left(\tilde{v} \mid \tilde{p}, \tilde{s}_{i}\right)-\operatorname{var}^{-1}(\tilde{v}-$ $\left.\tilde{p} \mid \tilde{s}_{i}\right)>0$. By equation (13) and by $\partial m / \partial \mu>0$, we know that $\partial \operatorname{var}^{-1}\left(\tilde{v} \mid \tilde{p}, \tilde{s}_{i}\right) / \partial \mu>0$. By equation (12),

$$
\frac{1}{\operatorname{var}\left(\tilde{v}-\tilde{p} \mid \tilde{s}_{i}\right)}=\frac{\mu^{2} \rho_{x}\left(\rho_{v}+\rho_{\varepsilon}\right)}{\gamma^{2}+\mu^{2} \rho_{x}\left(\rho_{v}+\rho_{\varepsilon}\right)} \frac{1}{\operatorname{var}\left(\tilde{v} \mid \tilde{p}, \tilde{s}_{i}\right)} .
$$

So, $\operatorname{var}^{-1}\left(\tilde{v}-\tilde{p} \mid \tilde{s}_{i}\right)$ is increasing in $\mu$ as well, because both $\mu^{2} \rho_{x}\left(\rho_{v}+\rho_{\varepsilon}\right) /\left[\gamma^{2}+\mu^{2} \rho_{x}\left(\rho_{v}+\right.\right.$ $\left.\left.\rho_{\varepsilon}\right)\right]$ and $\operatorname{var}^{-1}\left(\tilde{v} \mid \tilde{p}, \tilde{s}_{i}\right)$ are increasing in $\mu$; that is, $\partial \operatorname{var}^{-1}\left(\tilde{v}-\tilde{p} \mid \tilde{s}_{i}\right) / \partial \mu>0$. Thus,

$$
\mu \frac{\partial}{\partial \mu} \frac{1}{\operatorname{var}\left(\tilde{v} \mid \tilde{p}, \tilde{s}_{i}\right)}+(1-\mu) \frac{\partial}{\partial \mu} \frac{1}{\operatorname{var}\left(\tilde{v}-\tilde{p} \mid \tilde{s}_{i}\right)}>0 .
$$

Proof of Corollary 3. By the variance decomposition formula,

$$
\operatorname{var}(\tilde{v}-\tilde{p})=\operatorname{var}\left(\tilde{v}-\tilde{p} \mid \tilde{s}_{i}\right)+\operatorname{var}\left[\mathrm{E}\left(\tilde{v}-\tilde{p} \mid \tilde{s}_{i}\right)\right] .
$$

We have shown:

$$
\frac{\partial \operatorname{var}\left(\tilde{v}-\tilde{p} \mid \tilde{s}_{i}\right)}{\partial \mu}<0
$$

Now examine $\partial \operatorname{var}\left[\mathrm{E}\left(\tilde{v}-\tilde{p} \mid \tilde{s}_{i}\right)\right] / \partial \mu$. Direct computation shows:

$$
\operatorname{var}\left[\mathrm{E}\left(\tilde{v}-\tilde{p} \mid \tilde{s}_{i}\right)\right]=(1-\beta)^{2} \frac{\rho_{\varepsilon}}{\left(\rho_{v}+\rho_{\varepsilon}\right) \rho_{v}} .
$$

By equation (9) in Proposition 1,

$$
(1-\beta)=\frac{\rho_{v}+\rho_{\varepsilon}-(\gamma / \mu) m}{\rho_{v}+\rho_{\varepsilon}+m^{2} \rho_{x}},
$$

and the definition of $m$ in Proposition 1 shows:

$$
\rho_{v}+\rho_{\varepsilon}-(\gamma / \mu) m=\rho_{v} \frac{\gamma^{2}+\mu^{2} \rho_{v} \rho_{x}+\mu^{2} \rho_{x} \rho_{\varepsilon}}{\gamma^{2}+\mu^{2} \rho_{v} \rho_{x}+\mu \rho_{x} \rho_{\varepsilon}}>0 .
$$

Therefore, $(1-\beta)>0$.

Direct computation shows:

$$
\begin{aligned}
\frac{\partial(1-\beta)}{\partial m} & =-\frac{(\gamma / \mu)\left(\rho_{v}+\rho_{\varepsilon}+m^{2} \rho_{x}\right)+\left(\rho_{v}+\rho_{\varepsilon}-(\gamma / \mu) m\right)\left(2 m \rho_{x}\right)}{\left(\rho_{v}+\rho_{\varepsilon}+m^{2} \rho_{x}\right)^{2}} \\
& <0,
\end{aligned}
$$

because $(\gamma / \mu)\left(\rho_{v}+\rho_{\varepsilon}+m^{2} \rho_{x}\right)>0$ and $\left(\rho_{v}+\rho_{\varepsilon}-(\gamma / \mu) m\right)>0$.

Thus,

$$
\frac{\partial \operatorname{var}\left[\mathrm{E}\left(\tilde{v}-\tilde{p} \mid \tilde{s}_{i}\right)\right]}{\partial \mu}=2(1-\beta) \frac{\rho_{\varepsilon}}{\left(\rho_{v}+\rho_{\varepsilon}\right) \rho_{v}} \frac{\partial(1-\beta)}{\partial m} \frac{\partial m}{\partial \mu}<0
$$


and hence

$$
\frac{\partial \operatorname{var}(\tilde{v}-\tilde{p})}{\partial \mu}=\frac{\partial \operatorname{var}\left(\tilde{v}-\tilde{p} \mid \tilde{s}_{i}\right)}{\partial \mu}+\frac{\partial \operatorname{var}\left[\mathrm{E}\left(\tilde{v}-\tilde{p} \mid \tilde{s}_{i}\right)\right]}{\partial \mu}<0 .
$$

Proof of Corollary 4. Direct computation shows:

(A-1) $\frac{\partial(1 / \lambda)}{\partial \mu}=\frac{\left[2 m(\gamma / \mu)+m^{2} \rho_{x}-\left(\rho_{v}+\rho_{\varepsilon}\right)\right] \rho_{x} \frac{\partial m}{\partial \mu}+\frac{\gamma}{\mu^{2}}\left(\rho_{v}+\rho_{\varepsilon}+m^{2} \rho_{x}\right)}{\left((\gamma / \mu)+m \rho_{x}\right)^{2}}$.

Thus, $\partial(1 / \lambda) / \partial \mu>0$ if and only if $\left[2 m(\gamma / \mu)+m^{2} \rho_{x}-\left(\rho_{v}+\rho_{\varepsilon}\right)\right] \rho_{x}(\partial m / \partial \mu)+\gamma \mu^{-2}\left(\rho_{v}+\right.$ $\left.\rho_{\varepsilon}+m^{2} \rho_{x}\right)>0$. By equations (11) and (14), we can show that this is true:

$$
\begin{aligned}
{[2 m(\gamma / \mu)+} & \left.m^{2} \rho_{x}-\left(\rho_{v}+\rho_{\varepsilon}\right)\right] \rho_{x}(\partial m / \partial \mu)+\gamma \mu^{-2}\left(\rho_{v}+\rho_{\varepsilon}+m^{2} \rho_{x}\right) \\
= & \frac{\left(\gamma^{2}+\mu^{2} \rho_{v} \rho_{x}+\mu^{2} \rho_{x} \rho_{\varepsilon}\right)^{2}}{\gamma^{3} \mu^{2}\left(\gamma^{2}+\mu^{2} \rho_{v} \rho_{x}+\mu \rho_{x} \rho_{\varepsilon}\right)^{4}} \\
& \times\left[\begin{array}{c}
\left(\rho_{v}+\rho_{\varepsilon}\right) \gamma^{8}+\mu \rho_{x}\left(4 \rho_{\varepsilon}^{2}+\rho_{v} \rho_{\varepsilon}(4-\mu)+2 \mu \rho_{v}^{2}\right) \gamma^{6} \\
+\mu^{2} \rho_{x}^{2}\left(6 \rho_{\varepsilon}^{3}+\mu^{2} \rho_{v}^{3}+2(3-\mu) \rho_{v} \rho_{\varepsilon}^{2}+2 \mu \rho_{v}^{2} \rho_{\varepsilon}\right) \gamma^{4} \\
+\mu^{3} \rho_{x}^{3} \rho_{\varepsilon}^{3}\left((4-\mu) \rho_{v}+4 \rho_{\varepsilon}\right) \gamma^{2}+\left(\mu^{4} \rho_{x}^{4} \rho_{\varepsilon}^{5}+\rho_{v} \mu^{4} \rho_{x}^{4} \rho_{\varepsilon}^{4}\right)
\end{array}\right]>0 .
\end{aligned}
$$

Proof of Proposition 2. The derivative of the exchange's profit with respect to $\mu$ is

$$
\pi^{\prime}(\mu)=\frac{1}{2 \gamma}\left(\ln \left[1+\frac{\gamma^{2}}{\mu^{2} \rho_{x}\left(\rho_{v}+\rho_{\varepsilon}\right)}\right]-\frac{2}{1+\frac{\gamma^{2}}{\mu^{2} \rho_{x}\left(\rho_{v}+\rho_{\varepsilon}\right)}} \frac{\gamma^{2}}{\mu^{2} \rho_{x}\left(\rho_{v}+\rho_{\varepsilon}\right)}\right) .
$$

Define

$$
z(\mu) \equiv \frac{\gamma^{2}}{\mu^{2} \rho_{x}\left(\rho_{v}+\rho_{\varepsilon}\right)} \in\left[\frac{\gamma^{2}}{\rho_{x}\left(\rho_{v}+\rho_{\varepsilon}\right)}, \infty\right),
$$

and its inverse function is

$$
\mu(z) \equiv \frac{\gamma}{\sqrt{z \rho_{x}\left(\rho_{v}+\rho_{\varepsilon}\right)}} .
$$

So, we have

$$
\pi^{\prime}(\mu)=\frac{1}{2 \gamma}\left[\ln (1+z(\mu))-\frac{2 z(\mu)}{1+z(\mu)}\right] .
$$

Now we will examine the properties of function $f(z)=\ln (1+z)-(2 z) /(1+z)$. We are going to show that i) there is a unique positive solution $\hat{z} \in(0, \infty)$ that solves the equation $f(\hat{z})=0$, and that ii) for $z \in(0, \hat{z})$, we have $f(z)<0$, and for $z \in(\hat{z}, \infty)$, we have $f(z)>0$. Specifically, because $z$ is close to 0 , by first-order Taylor expansion, $f(z) \approx$ $z-2 z=-z<0$, and when $z$ is large, $\lim _{z \rightarrow \infty} f(z)=\infty$. Thus, there will be a $\hat{z} \in(0, \infty)$ such that $f(\hat{z})=0$. Numerically solving shows that $\hat{z} \approx 3.92$. Taking the first-order derivative of $f(z)$ delivers

$$
f^{\prime}(z)=\frac{z-1}{(1+z)^{2}} .
$$

So for $z<1, f(z)$ is decreasing and negative because when $z$ is small, $f(z)$ is negative. Then, as we increase $z$ above $1, f(z)$ will be increasing, and it will cross 0 once and then stay positive. Thus, we know that as long $0<z<\hat{z}$, then $f(z)<0$, and when $z>\hat{z}$, we have $f(z)>0$. 
So it is clear that if $\gamma^{2} /\left[\rho_{x}\left(\rho_{v}+\rho_{\varepsilon}\right)\right]>\hat{z}$, then we must have $\pi^{\prime}(\mu)>0$ for all $\mu \epsilon$ $[0,1]$, because for the whole range of $z(\mu) \in\left[\gamma^{2} /\left[\rho_{x}\left(\rho_{v}+\rho_{\varepsilon}\right)\right], \infty\right), z$ is greater than $\hat{z}$, and as a result $\pi^{\prime}(\mu)=f(z(\mu))>0$, and the maximum profit of the exchange is achieved at $\mu^{*}=1$. If $\gamma^{2} /\left[\rho_{x}\left(\rho_{v}+\rho_{\varepsilon}\right)\right]<\hat{z}$, then for $\mu \in(0, \mu(\hat{z}))$, we know $z(\mu) \in(\hat{z}, \infty)$, and hence $\pi^{\prime}(\mu)=f(z(\mu))>0$; and for $\mu \in(\mu(\hat{z}), 1)$, we have $z(\mu) \in\left(\gamma^{2} /\left[\rho_{x}\left(\rho_{v}+\rho_{\varepsilon}\right)\right], \hat{z}\right)$, and hence $\pi^{\prime}(\mu)=f(z(\mu))<0$. So, the maximum is achieved at $\mu^{*}=\mu(\hat{z})=\gamma / \sqrt{\hat{z} \rho_{x}\left(\rho_{v}+\rho_{\varepsilon}\right)}$. The optimal price $q^{*}$ can be computed by substituting $\mu^{*}$ into the definition of $B(\mu)$ and using the condition of $f(\hat{z})=0$.

Proof of Proposition 5. We first prove the following lemma, which provides a range for $m$ and an expression for $(1-\beta)$.

Lemma $M$. In equilibrium, we have:

$$
\begin{aligned}
\frac{\mu_{p s} \rho_{\varepsilon}}{\gamma} \leq m<\frac{\mu_{p s} \rho_{\varepsilon}+\left(\mu_{p s}+\mu_{p}\right) \rho_{v}}{\gamma}, \text { and } \\
1-\beta=\frac{\mu_{p s} \rho_{\varepsilon}+\left(\mu_{p s}+\mu_{p}\right) \rho_{v}-\gamma m}{\mu_{p s} \rho_{\varepsilon}+\left(\mu_{p s}+\mu_{p}\right)\left(\rho_{v}+m^{2} \rho_{x}\right)}>0 .
\end{aligned}
$$

Proof. When $\mu_{s}=0$, by Corollary 6, we have $m=\gamma^{-1} \mu_{p s} \rho_{\varepsilon}$. Now suppose $\mu_{s}>0$. We can rewrite $L(m)=0$ as:

$$
\begin{aligned}
\frac{\gamma m}{\rho_{\varepsilon}}= & \mu_{p s}+\mu_{s} \\
& \times \frac{\left[\mu_{p s} \rho_{\varepsilon}+\left(\mu_{p s}+\mu_{p}\right) \rho_{v}-\gamma m\right]\left[\mu_{p s} \rho_{\varepsilon}+\left(\mu_{p s}+\mu_{p}\right)\left(\rho_{v}+m^{2} \rho_{x}\right)\right]}{\left[\mu_{p s} \rho_{\varepsilon}+\left(\mu_{p s}+\mu_{p}\right) \rho_{v}-\gamma m\right]^{2}+\left[\gamma+\left(\mu_{p s}+\mu_{p}\right) m \rho_{x}\right]^{2} \frac{\rho_{v}+\rho_{\varepsilon}}{\rho_{x}}} .
\end{aligned}
$$

We consider two possible cases depending on the sign of $\left[\mu_{p s} \rho_{\varepsilon}+\left(\mu_{p s}+\mu_{p}\right) \rho_{v}-\gamma m\right]$. If $\mu_{p s} \rho_{\varepsilon}+\left(\mu_{p s}+\mu_{p}\right) \rho_{v}-\gamma m \leq 0$, or, equivalently, if

$$
\gamma m \geq \mu_{p s} \rho_{\varepsilon}+\left(\mu_{p s}+\mu_{p}\right) \rho_{v},
$$

then the second term in the right-hand side of equation (A-4) is negative, and hence by (A-4), we have:

$$
\frac{\gamma m}{\rho_{\varepsilon}}<\mu_{p s} \Leftrightarrow \gamma m \leq \mu_{p s} \rho_{\varepsilon} .
$$

This contradicts the condition in equation (A-5). So, in equilibrium, we must have $\gamma m / \rho_{\varepsilon} \geq \mu_{p s}$ and $\gamma m<\mu_{p s} \rho_{\varepsilon}+\left(\mu_{p s}+\mu_{p}\right) \rho_{v}$, which imply the condition in equation (A-2). By the expression of $\beta$ in equation (17), we can compute $1-\beta=\left[\mu_{p s} \rho_{\varepsilon}+\left(\mu_{p s}+\mu_{p}\right)\right.$ $\left.\rho_{v}-\gamma m\right] /\left[\mu_{p s} \rho_{\varepsilon}+\left(\mu_{p s}+\mu_{p}\right)\left(\rho_{v}+m^{2} \rho_{x}\right)\right]$. Given $\gamma m<\mu_{p s} \rho_{\varepsilon}+\left(\mu_{p s}+\mu_{p}\right) \rho_{v}$, we know $(1-\beta)>0$.

We now prove Proposition 5. By the expressions of $\phi$ in equations (26)-(28), we can compute:

$$
e^{2 \gamma\left(\phi_{p s}-\phi_{s}-\phi_{p}\right)}-1=\frac{\lambda^{2} \rho_{\varepsilon} \rho_{v}\left(\rho_{v}+\rho_{\varepsilon}\right) H\left(m, \mu_{p s}, \mu_{p}\right)}{\left(\rho_{x}(1-\beta)^{2}+\lambda^{2} \rho_{v}\right)\left(\rho_{v}+m^{2} \rho_{x}\right)\left(\rho_{v}+\rho_{\varepsilon}\right)},
$$

where

$$
H\left(m, \mu_{p s}, \mu_{p}\right) \equiv 1-m^{2} \frac{\rho_{x}^{2}}{\rho_{v}\left(\rho_{v}+\rho_{\varepsilon}\right)}\left(\frac{1-\beta}{\lambda}\right)^{2} .
$$

So,

$$
\begin{aligned}
\phi_{p s} & >\phi_{p}+\phi_{s} \Leftrightarrow H\left(m, \mu_{p s}, \mu_{p}\right) \\
& >0 \Leftrightarrow m \frac{\rho_{x}}{\sqrt{\rho_{v}\left(\rho_{v}+\rho_{\varepsilon}\right)}} \frac{1-\beta}{\lambda}<1,
\end{aligned}
$$


where the last arrow in equation (A-8) follows from the expression of $H\left(m, \mu_{p s}, \mu_{p}\right)$ and the fact that $(1-\beta)>0$ in equation (A-3).

Suppose $\phi_{p s} \leq \phi_{p}+\phi_{s}$; that is, the two signals $\tilde{p}$ and $\tilde{s}_{i}$ are substitutes. Then, by the condition in equation (A-8), we must have $\left[m \rho_{x}(1-\beta)\right] /\left[\gamma \sqrt{\rho_{v}\left(\rho_{v}+\rho_{\varepsilon}\right)}\right] \geq 1$. By the expressions of $(1-\beta)$ in equation (A-3) and $\lambda$ in equation (18), we can show:

$$
m \frac{\rho_{x}}{\sqrt{\rho_{v}\left(\rho_{v}+\rho_{\varepsilon}\right)}} \frac{1-\beta}{\lambda} \geq 1 \Leftrightarrow \gamma m^{2}+C_{1} m+C_{0} \leq 0,
$$

where

$$
\begin{aligned}
C_{1} & \equiv \sqrt{\rho_{v}\left(\rho_{v}+\rho_{\varepsilon}\right)}\left(\mu_{p s}+\mu_{p}\right)-\left(\mu_{p s} \rho_{\varepsilon}+\left(\mu_{p s}+\mu_{p}\right) \rho_{v}\right), \\
C_{0} & \equiv \frac{\gamma \sqrt{\rho_{v}\left(\rho_{v}+\rho_{\varepsilon}\right)}}{\rho_{x}} .
\end{aligned}
$$

An information market equilibrium is supported (i.e., the condition in equation (A-9) holds for some positive value of $m$; note that $m>0$ by equation (A-2) in Lemma M) only if the following two conditions are satisfied simultaneously:

$$
C_{1}<0 \text { and } C_{1}^{2} \geq 4 \gamma C_{0} .
$$

Under these two conditions, the condition in equation (A-9) holds for

$$
m \in\left[\frac{-C_{1}-\sqrt{C_{1}^{2}-4 \gamma C_{0}}}{2 \gamma}, \frac{-C_{1}+\sqrt{C_{1}^{2}-4 \gamma C_{0}}}{2 \gamma}\right] .
$$

However, we can show that $(2 \gamma)^{-1}\left(-C_{1}+\sqrt{C_{1}^{2}-4 \gamma C_{0}}\right)<\gamma^{-1} \rho_{\varepsilon} \mu_{p s}$, which means $m<\gamma^{-1} \rho_{\varepsilon} \mu_{p s}$, contradicting the condition in equation (A-2) in Lemma M. To see this, note that

$$
\begin{aligned}
\rho_{v} & <\sqrt{\rho_{v}\left(\rho_{v}+\rho_{\varepsilon}\right)} \Rightarrow\left(\mu_{p s}+\mu_{p}\right) \rho_{v}<\sqrt{\rho_{v}\left(\rho_{v}+\rho_{\varepsilon}\right)}\left(\mu_{p s}+\mu_{p}\right) \\
& \Rightarrow-\sqrt{\rho_{v}\left(\rho_{v}+\rho_{\varepsilon}\right)}\left(\mu_{p s}+\mu_{p}\right)+\left(\mu_{p s} \rho_{\varepsilon}+\left(\mu_{p s}+\mu_{p}\right) \rho_{v}\right)<\rho_{\varepsilon} \mu_{p s} \\
& \Rightarrow-C_{1}<\rho_{\varepsilon} \mu_{p s},
\end{aligned}
$$

where the last inequality follows from the definition of $C_{1}$ in equation (A-10). So, given $-C_{1}>0$ and $C_{1}^{2} \geq 4 \gamma C_{0}$ in (A-12), we have:

$$
\frac{-C_{1} \pm \sqrt{C_{1}^{2}-4 \gamma C_{0}}}{2 \gamma} \leq \frac{-C_{1} \pm \sqrt{C_{1}^{2}}}{2 \gamma} \leq-\frac{C_{1}}{\gamma}<\frac{\rho_{\varepsilon} \mu_{p s}}{\gamma} .
$$

Proof of Corollary 5. We provide a proof by contradiction. Specifically, suppose $\mu_{p}>0$ and $\mu_{s}>0$; we then show that $\phi_{p s} \leq \phi_{p}+\phi_{s}$ in equilibrium, contradicting Proposition 5. There are four possible cases depending on whether $\mu_{p s}$ and $\mu_{u}$ are 0 or positive.

Case 1. $\mu_{p s}>0$ and $\mu_{u}>0$. In this case, we must have $\phi_{p s}-q-c=\phi_{p}-q=\phi_{s}-c=0$. So, $\phi_{p s}=q+c, \phi_{p}=q$ and $\phi_{s}=c$, which jointly imply $\phi_{p s}=\phi_{p}+\phi_{s}$.

Case 2. $\mu_{p s}>0$ and $\mu_{u}=0$. In this case, $\phi_{p s}-q-c=\phi_{p}-q=\phi_{s}-c \geq 0$. If $\phi_{p s}-q-c=$ $\phi_{p}-q=\phi_{s}-c=0$, then $\phi_{p s}=\phi_{p}+\phi_{s}$; and if $\phi_{p s}-q-c=\phi_{p}-q=\phi_{s}-c>0$, then $\phi_{p s}<$ $\phi_{p}+\phi_{s}$.

Case 3. $\mu_{p s}=0$ and $\mu_{u}>0$. In this case, $\phi_{p s}-q-c \leq \phi_{p}-q=\phi_{s}-c=0$. So, $\phi_{p s} \leq q+c$, $\phi_{p}=q$ and $\phi_{s}=c$, which jointly imply $\phi_{p s} \leq \phi_{p}+\phi_{s}$. 
Case 4. $\mu_{p s}=0$ and $\mu_{u}=0$. In this case, $\phi_{p}-q=\phi_{s}-c, \phi_{p s}-q-c \leq \phi_{s}-c$, and $\phi_{s}-c \geq 0$. So, the first two conditions say: $\phi_{p}-q=\phi_{s}-c \Rightarrow q=\phi_{p}-\phi_{s}+c$ and $\phi_{p s}-q-c \leq \phi_{s}-$ $c \Rightarrow \phi_{p s}-q \leq \phi_{s}$. They jointly imply $\phi_{p s}-\left(\phi_{p}-\phi_{s}+c\right) \leq \phi_{s} \Rightarrow \phi_{p s}-\phi_{p} \leq c$. However, $\phi_{s}-$ $c \geq 0 \Rightarrow \phi_{s} \geq c$. So, we have $\phi_{p s}-\phi_{p} \leq \phi_{s}$, which implies $\phi_{p s} \leq \phi_{p}+\phi_{s}$.

Proof of Propositions 6 and 8. The existence part of Proposition 6 follows from Part (a) of Lemmas 1-8 in the Internet Appendix. Specifically, the union of the sets of $(q, c)$ characterizing each type of information allocation covers $\mathbb{R}_{++}^{2}$. Thus, for any given information price pair $(q, c) \in \mathbb{R}_{++}^{2}$, there is an information allocation supported by this price pair. The uniqueness part of Proposition 6 follows from Part (b) of Lemmas 1-8 and from the fact that the $(q, c)$ sets in Part (a) are mutually exclusive. That is, for a given price pair $(q, c) \in \mathbb{R}_{++}^{2}$, because the $(q, c)$ sets in Part (a) are mutually exclusive, one can determine a particular trader distribution. In addition, Part (b) shows that given this trader distribution, there exists a unique information allocation equilibrium supported by the price pair $(q, c)$.

Now we prove Proposition 8. If $c \leq \underline{c}$, then $\mu_{p s}^{F}=1$ in ECONOMY ${ }^{\mathrm{F}}$. Note that the upper boundary of Case (1) in Lemma 1 of the Internet Appendix is downward sloping and approaches 0 as $q$ goes to $\infty$ and that the upper boundary of Case (5) in Lemma 5 is upward sloping and approaches $\bar{c}$ as $q$ goes to $\infty$. So, when $q$ becomes sufficiently large, the information allocation will eventually fall into Case (5) with $\mu_{p s}>0, \mu_{u}>0$, and $\mu_{p}=\mu_{s}=0$. Thus, the total amount of fundamental information $\mu_{p s}$ will become lower than that in ECONOMYF. If $\underline{c}<c<\bar{c}$, then $\mu_{p s}^{F}<1$ in ECONOMYF. Also, as $q$ becomes sufficiently large, the information allocation will eventually fall into Case (5), and Lemma 5 also shows that $\mu_{p s}<\mu_{p s}^{F}$.

Proof of Proposition 9. Given the conditions in Proposition 9, as $q$ increases from 0 to $\infty$, the equilibrium information allocation gradually moves from Case (4) to Case (3) and to Case (5) in ECONOMY ${ }^{\mathrm{D}}$ (see Figure 3). We prove Proposition 9 by examining the implications of $q$ in each case.

Case (4). In Case (4), only PS- and P-informed traders are active. By Lemma 4 in the Internet Appendix, we know that the equilibrium fraction $\mu_{p s}$ of PS-informed traders is fixed at the value of $\mu_{p s}^{F}$ given by Proposition 7. So, by Corollary 6, price informativeness is fixed at a constant of $m=\gamma^{-1} \mu_{p s}^{F} \rho_{\varepsilon}$ in this case. The equilibrium price coefficients are accordingly maintained at constants given by equations (17) and (18). The positive variables do not vary with $q$, given that the price functions do not change.

Case (3). In Case (3), PS-informed, P-informed, and uninformed traders are active. By Lemma 3 in the Internet Appendix, $\mu_{p s}$ is still fixed at a constant of $\mu_{p s}^{F}$, and the price informativeness is accordingly fixed at a constant of $m=\gamma^{-1} \mu_{p s}^{F} \rho_{\varepsilon}$. The proof of Lemma 3 of the Internet Appendix also shows that increasing $q$ will decrease $\mu_{p}$; that is, $\partial \mu_{p} / \partial q<0$ in Case (3).

By equation (18) and the facts that $\mu_{p s}=\mu_{p s}^{F}$ and $m=\gamma^{-1} \mu_{p s}^{F} \rho_{\varepsilon}$, we have

$$
\begin{aligned}
\lambda & =\frac{\gamma+\left(\mu_{p s}^{F}+\mu_{p}\right)\left(\frac{\mu_{p s}^{F} \rho_{\varepsilon}}{\gamma}\right) \rho_{x}}{\mu_{p s}^{F} \rho_{\varepsilon}+\left(\mu_{p s}^{F}+\mu_{p}\right)\left(\rho_{v}+\left(\frac{\mu_{p s}^{F} \rho_{\varepsilon}}{\gamma}\right)^{2} \rho_{x}\right)} \Rightarrow \\
\frac{\partial \lambda}{\partial \mu_{p}} & =\frac{-\gamma \rho_{v}}{\left[\mu_{p s}^{F} \rho_{\varepsilon}+\left(\mu_{p s}^{F}+\mu_{p}\right)\left(\rho_{v}+\left(\frac{\mu_{p s}^{F} \rho_{\varepsilon}}{\gamma}\right)^{2} \rho_{x}\right)\right]^{2}}<0 .
\end{aligned}
$$

So, increasing $q$ will decrease liquidity through decreasing $\mu_{p}$ in Case (3). 

tion shows:

Using the fact that $\beta=m \lambda=\gamma^{-1} \mu_{p s}^{F} \rho_{\varepsilon} \lambda$ and equations (17) and (18), direct computa-

$$
\begin{aligned}
\frac{\partial \operatorname{var}(\tilde{v}-\tilde{p})}{\partial \mu_{p}} & =2\left[(\beta-1) \frac{1}{\rho_{v}} \frac{\mu_{p s}^{F} \rho_{\varepsilon}}{\gamma}+\lambda \frac{1}{\rho_{x}}\right] \frac{\partial \lambda}{\partial \mu_{p}} \\
& =\frac{2 \gamma^{3}}{\rho_{x}\left[\gamma^{2} \mu_{p} \rho_{v}+\gamma^{2} \mu_{p s}^{F}\left(\rho_{v}+\rho_{\varepsilon}\right)+\left(\mu_{p}+\mu_{p s}^{F}\right)\left(\mu_{p s}^{F}\right)^{2} \rho_{\varepsilon}^{2} \rho_{x}\right]} \frac{\partial \lambda}{\partial \mu_{p}} \\
& <0 .
\end{aligned}
$$

So, increasing $q$ will also increase return volatility through decreasing $\mu_{p}$.

The cost of capital is negatively related to the average precision across all investors, that is:

$$
\frac{\mu_{p s}^{F}}{\operatorname{var}\left(\tilde{v}-\tilde{p} \mid \tilde{p}, \tilde{s}_{i}\right)}+\frac{\mu_{p}}{\operatorname{var}(\tilde{v}-\tilde{p} \mid \tilde{p})}+\frac{1-\mu_{p s}^{F}-\mu_{p}}{\operatorname{var}(\tilde{v}-\tilde{p})} .
$$

By equations (22) and (23) and the fact that $m=\gamma^{-1} \mu_{p s}^{F} \rho_{\varepsilon}$, we know that increasing $q$ will

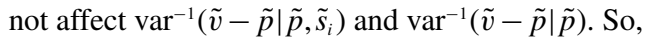

$$
\begin{aligned}
\frac{\partial}{\partial q}[ & \left.\frac{\mu_{p s}^{F}}{\operatorname{var}\left(\tilde{v}-\tilde{p} \mid \tilde{p}, \tilde{s}_{i}\right)}+\frac{\mu_{p}}{\operatorname{var}(\tilde{v}-\tilde{p} \mid \tilde{p})}+\frac{1-\mu_{p s}^{F}-\mu_{p}}{\operatorname{var}(\tilde{v}-\tilde{p})}\right] \\
& =\frac{\partial}{\partial \mu_{p}}\left[\frac{\mu_{p}}{\operatorname{var}(\tilde{v}-\tilde{p} \mid \tilde{p})}+\frac{1-\mu_{p s}^{F}-\mu_{p}}{\operatorname{var}(\tilde{v}-\tilde{p})}\right] \frac{\partial \mu_{p}}{\partial q} \\
& =\left[\left(\frac{1}{\operatorname{var}(\tilde{v}-\tilde{p} \mid \tilde{p})}-\frac{1}{\operatorname{var}(\tilde{v}-\tilde{p})}\right)+\left(1-\mu_{p s}^{F}-\mu_{p}\right) \frac{\partial \frac{1}{\operatorname{var}(\tilde{v}-\tilde{p})}}{\partial \mu_{p}}\right] \frac{\partial \mu_{p}}{\partial q} \\
& <0
\end{aligned}
$$

because $\operatorname{var}^{-1}(\tilde{v}-\tilde{p} \mid \tilde{p})-\operatorname{var}^{-1}(\tilde{v}-\tilde{p})>0$ (P-informed traders know more than uninformed traders), $\partial \operatorname{var}^{-1}(\tilde{v}-\tilde{p}) / \partial \mu_{p}>0$, and $\partial \mu_{p} / \partial q<0$. Therefore, we have $\partial C C / \partial q>0$; that is, increasing $q$ will increase the cost of capital. The implication for liquidity traders' welfare follows immediately from the implications for liquidity and the cost of capital.

Case (5). In Case (5), only PS-informed and uninformed traders are active. In Lemma 5 of the Internet Appendix, we show that $\mu_{p s}$ is determined by

$$
\phi_{p s}=\frac{1}{2 \gamma} \ln \left[1+\frac{\gamma^{2}\left(\gamma^{2}+\mu_{p s}^{2} \rho_{\varepsilon} \rho_{x}\right)}{\left[\gamma^{2}\left(\rho_{v}+\rho_{\varepsilon}\right)+\mu_{p s}^{2} \rho_{\varepsilon}^{2} \rho_{x}\right] \mu_{p s}^{2} \rho_{x}}\right]=q+c .
$$

Direct computation shows:

$$
\begin{aligned}
\frac{\partial \phi_{p s}}{\partial \mu_{p s}^{2}} & =\frac{\partial}{\partial \mu_{p s}^{2}} \frac{\gamma^{2}\left(\gamma^{2}+\mu_{p s}^{2} \rho_{\varepsilon} \rho_{x}\right)}{\left[\gamma^{2}\left(\rho_{v}+\rho_{\varepsilon}\right)+\mu_{p s}^{2} \rho_{\varepsilon}^{2} \rho_{x}\right] \mu_{p s}^{2} \rho_{x}} \\
& =-\frac{\gamma^{2}\left(\gamma^{4} \rho_{v}+\gamma^{4} \rho_{\varepsilon}+2 \gamma^{2} \mu_{p s}^{2} \rho_{\varepsilon}^{2} \rho_{x}+\mu_{p s}^{4} \rho_{\varepsilon}^{3} \rho_{x}^{2}\right)}{\rho_{x}\left[\left(\gamma^{2} \rho_{v}+\gamma^{2} \rho_{\varepsilon}+\mu_{p s}^{2} \rho_{\varepsilon}^{2} \rho_{x}\right) \mu_{p s}^{2}\right]^{2}}<0 .
\end{aligned}
$$

Thus, we have $\partial \mu_{p s} / \partial q<0$ by the implicit function theorem.

Because $\mu_{s}=0$, by Corollary 6, price informativeness is still given by $m=\gamma^{-1} \mu_{p s} \rho_{\varepsilon}$. So, $\partial m / \partial q=\gamma^{-1} \rho_{\varepsilon} \partial \mu_{p s} / \partial q<0$. 
By equation (18) in Proposition 4, and that fact that $\mu_{p}=0$ and $m=\gamma^{-1} \mu_{p s} \rho_{\varepsilon}$, we can express $\lambda$ as a function of $\mu_{p s}$ only:

$$
\lambda=\frac{\gamma+\mu_{p s} \frac{\mu_{p s} \rho_{\varepsilon}}{\gamma} \rho_{x}}{\mu_{p s}\left[\rho_{v}+\rho_{\varepsilon}+\left(\frac{\mu_{p s} \rho_{\varepsilon}}{\gamma}\right)^{2} \rho_{x}\right]} .
$$

Taking the direct derivative with respect to $\mu_{p s}$ shows:

$$
\frac{\partial \lambda}{\partial \mu_{p s}}=-\frac{\gamma^{4}\left(\rho_{v}+\rho_{\varepsilon}+\rho_{v} \mu_{p s}\right)+\gamma^{2} \mu_{p s}^{2} \rho_{\varepsilon} \rho_{x}\left(-\rho_{v}+2 \rho_{\varepsilon}+\mu_{p s} \rho_{v}\right)+\mu_{p s}^{4} \rho_{\varepsilon}^{3} \rho_{x}^{2}}{\gamma^{3} \mu_{p s}^{2}\left[\rho_{\varepsilon}+\rho_{v}+\left(\frac{\mu_{p s} \rho_{\varepsilon}}{\gamma}\right)^{2} \rho_{x}\right]^{2}}
$$

So, when $\gamma$ is large (as in Proposition 9), we have $\partial \lambda / \partial \mu_{p s}<0$; that is, increasing $q$ will harm liquidity, $\lambda^{-1}$, through decreasing $\mu_{p s}$ (i.e., $\partial(1 / \lambda) / \partial q=-\lambda^{-2}\left(\partial \lambda / \partial \mu_{p s}\right)$ $\left.\left(\partial \mu_{p s} / \partial q\right)<0\right)$.

By

$$
\frac{\partial \phi_{p s}}{\partial \mu_{p s}}=\frac{\partial}{\partial \mu_{p s}}\left(\frac{1}{2 \gamma} \ln \left[\frac{\operatorname{var}(\tilde{v}-\tilde{p})}{\operatorname{var}\left(\tilde{v}-\tilde{p} \mid \tilde{s}_{i}\right)}\right]\right)<0,
$$

we know $\partial\left[\operatorname{var}(\tilde{v}-\tilde{p}) / \operatorname{var}\left(\tilde{v}-\tilde{p} \mid \tilde{p}, \tilde{s}_{i}\right)\right] / \partial \mu_{p s}<0$. By equation (22) and $m=\gamma^{-1} \mu_{p s} \rho_{\varepsilon}$, we have $\partial \operatorname{var}^{-1}\left(\tilde{v}-\tilde{p} \mid \tilde{p}, \tilde{s}_{i}\right) / \partial \mu_{p s}>0$. So it must be the case that $\partial \operatorname{var}(\tilde{v}-\tilde{p}) / \partial \mu_{p s}<0$. Because increasing $q$ will decrease $\mu_{p s}, \operatorname{var}(\tilde{v}-\tilde{p})$ increases with $q$.

The cost of capital will increase with $q$ as well. Recall that by Proposition 4 , the cost of capital is negatively related to the average precision of traders' forecasts:

$$
\frac{\mu_{p s}}{\operatorname{var}\left(\tilde{v}-\tilde{p} \mid \tilde{p}, \tilde{s}_{i}\right)}+\frac{1-\mu_{p s}}{\operatorname{var}(\tilde{v}-\tilde{p})} .
$$

Clearly,

$$
\begin{aligned}
\frac{\partial}{\partial \mu_{p s}}[ & \left.\frac{\mu_{p s}}{\operatorname{var}\left(\tilde{v}-\tilde{p} \mid \tilde{p}, \tilde{s}_{i}\right)}+\frac{1-\mu_{p s}}{\operatorname{var}(\tilde{v}-\tilde{p})}\right] \\
= & {\left[\frac{1}{\operatorname{var}\left(\tilde{v}-\tilde{p} \mid \tilde{p}, \tilde{s}_{i}\right)}-\frac{1}{\operatorname{var}(\tilde{v}-\tilde{p})}\right] } \\
& +\mu_{p s} \frac{\partial}{\partial \mu_{p s}} \frac{1}{\operatorname{var}\left(\tilde{v}-\tilde{p} \mid \tilde{p}, \tilde{s}_{i}\right)}+\left(1-\mu_{p s}\right) \frac{\partial}{\partial \mu_{p s}} \frac{1}{\operatorname{var}(\tilde{v}-\tilde{p})}>0 .
\end{aligned}
$$

So, $\partial C C / \partial \mu_{p s}<0$. Given that increasing $q$ will decrease $\mu_{p s}$, we know that CC increases with $q$. Again, the implication for liquidity traders' welfare follows immediately from the implications for liquidity and the cost of capital.

\section{References}

Admati, A., and P. Pfleiderer. “A Monopolistic Market for Information.” Journal of Economic Theory, 39 (1986), 400-438.

Admati, A., and P. Pfleiderer. "Viable Allocations of Information in Financial Markets." Journal of Economic Theory, 43 (1987), 76-115.

Admati, A., and P. Pfleiderer. "Selling and Trading on Information in Financial Markets." American Economic Review, 78 (1988), 96-103.

Admati, A., and P. Pfleiderer. "Direct and Indirect Sale of Information." Econometrica, 58 (1990), 901-928. 
Allen, F. "The Market for Information and the Origin of Financial Intermediation." Journal of Financial Intermediation, 1 (1990), 3-30.

Arnuk, S., and J. Saluzzi. "Exchanges and Data Feeds: Data Theft on Wall Street." Themis Trading May 11 (2010).

Barber, B., and T. Odean. "Boys Will Be Boys: Gender, Overconfidence, and Common Stock Investment." Quarterly Journal of Economics, 116 (2001), 261-292.

Biais, B.; T. Foucault; and S. Moinas. "Equilibrium Fast Trading." Journal of Financial Economics, 116 (2015), 292-313.

Boehmer, E.; C. M. Jones; and X. Zhang. "Shackling Short Sellers: The 2008 Shorting Ban." Review of Financial Studies, 26 (2013), 1363-1400.

Boulatov, A., and M. Dierker. "Pricing Prices.” Working Paper, University of Houston (2007).

Brown, D. J., and G. M. Heal. "Marginal vs. Average Cost Pricing in the Presence of a Public Monopoly." American Economic Review (P\&P), 73 (1983), 189-193.

Cao, C.; L. C. Field; and G. Hanka. "Does Insider Trading Impair Market Liquidity? Evidence from IPO Lockup Expirations.” Journal of Financial and Quantitative Analysis, 39 (2004), 25-46.

Cespa, G., and T. Foucault. "Sale of Price Information by Exchanges: Does It Promote Price Discovery?” Management Science, 60 (2014), 148-165.

Chen, Z., and W. J. Wilhelm. "Sell-Side Information Production in Financial Markets." Journal of Financial and Quantitative Analysis, 47 (2012), 763-794.

Chowdhry, B., and V. Nanda. "Multimarket Trading and Market Liquidity." Review of Financial Studies, 4 (1991), 483-511.

Colliard, J.-E., and T. Foucault. "Trading Fees and Efficiency in Limit Order Markets." Review of Financial Studies, 25 (2012), 3305-3350.

Easley, D.; T. Hendershott; and T. Ramadorai. "Levelling the Trading Field." Journal of Financial Markets, 17 (2014), 65-93.

Easley, D., and M. O'Hara. "Microstructure and Ambiguity." Journal of Finance, 65 (2010), 1817-1846.

Fishman, M., and K. Hagerty. "The Optimal Amount of Discretion to Allow in Disclosure." Quarterly Journal of Economics, 105 (1990), 427-444.

Fishman, M., and K. Hagerty. "The Incentive to Sell Financial Market Information." Journal of Financial Intermediation, 4 (1995), 95-115.

Foucault, T., and T. Gehrig. "Stock Price Informativeness, Cross-Listings, and Investment Decisions." Journal of Financial Economics, 88 (2008), 146-168.

Foucault, T.; O. Kadan; and E. Kandel. "Liquidity Cycles and Make/Take Fees in Electronic Markets." Journal of Finance, 68 (2013), 299-341.

García, D., and F. Sangiorgi. "Information Sales and Strategic Trading." Review of Financial Studies, 24 (2011), 3069-3104.

García, D., and J. Vanden. "Information Acquisition and Mutual Funds.” Journal of Economic Theory, 144 (2009), 1965-1995.

Gennotte, G., and H. E. Leland. "Market Liquidity, Hedging, and Crashes." American Economic Review, 80 (1990), 999-1021.

Glosten, L. "Insider Trading, Liquidity and the Role of the Monopolist Specialist." Journal of Business, 62 (1989), 211-235.

Goldstein, I., and L. Yang. "Information Diversity and Complementarities in Trading and Information Acquisition." Journal of Finance, 70 (2015), 1723-1765.

Hasbrouck, J., and G. Saar. "Low-Latency Trading." Journal of Financial Markets, 16 (2013), 646-679.

Hendershott, T., and P. Moulton. “Automation, Speed, and Stock Market Quality: The NYSE's Hybrid." Journal of Financial Markets, 14 (2011), 568-604.

Judd, K. "The Law of Large Numbers with a Continuum of IID Random Variables." Journal of Economic Theory, 35 (1985), 19-25.

Kandel, E., and I. Tkatch. "Demand for the Immediacy of Execution: Time Is Money." Working Paper, Georgia State University and Hebrew University (2008).

Kyle, A. "Informed Speculation with Imperfect Competition.” Review of Economic Studies, 56 (1989), 317-356.

Leland, H. "Insider Trading: Should It Be Prohibited?” Journal of Political Economy, 100 (1992), $859-887$.

Odean, T. "Do Investors Trade Too Much?” American Economic Review, 89 (1999), 1279-1298.

O'Hara, M.; C. Yao; and M. Ye. "What's Not There: Odd Lots and Market Data." Journal of Finance, 69 (2014), 2199-2236.

Ozsoylev, H., and J. Walden. "Asset Pricing in Large Information Networks.” Journal of Economic Theory, 146 (2011), 2252-2280. 
Peress, J. "Product Market Competition, Insider Trading, and Stock Market Efficiency." Journal of Finance, 65 (2010), 1-43.

Peterson, K. "Speed Bump: NYSE Races to Cut Trading-Data Lag." Wall Street Journal, Dec. 1 (2010).

Subrahmanyam, A. "A Theory of Trading in Stock Index Futures." Review of Financial Studies, 4 (1991), 17-51.

Veldkamp, L. "Media Frenzies in Markets for Financial Information." American Economic Review, 96 (2006), 577-601.

Wang, Y. "Why Can Margin Requirements Increase Volatility and Benefit Margin Constrained Investors?" Review of Finance, 20 (2016), 1449-1485.

Zhu, H. “Do Dark Pools Harm Price Discovery?” Review of Financial Studies, 27 (2014), 747-789. 